\title{
Direct micro-seismic event location and characterization from passive seismic data using convolutional neural networks
}

\author{
Hanchen Wang* and Tariq Alkhalifah* \\ *Physical Sciences and Engineering Division, \\ King Abdullah University of Science and Technology, \\ 4700 KAUST,
} Thuwal, 23955, KSA

(Aug 13, 2020)

Running head: Direct micro-seismic locating with CNN 


\begin{abstract}
The ample size of time-lapse data often requires significant event detection and source location efforts, especially in areas like shale gas exploration regions where a large number of micro-seismic events are often recorded. In many cases, the real-time monitoring and locating of these events are essential to production decisions. Conventional methods face considerable drawbacks. For example, traveltime-based methods require traveltime picking of often noisy data, while migration and waveform inversion methods require expensive wavefield solutions and event detection. Both tasks require some human intervention, and this becomes a big problem when too many sources need to be located, which is common in micro-seismic monitoring. Machine learning has recently been used to identify microseismic events or locate their sources once they are identified and picked. We propose to use a novel artificial neural network framework to directly map seismic data, without any event picking or detection, to their potential source locations. We train two convolutional neural networks on labeled synthetic acoustic data containing simulated micro-seismic events to fulfill such requirements. One convolutional neural network, which has a global average pooling layer to reduce the computational cost while maintaining high-performance levels, aims to classify the number of events in the data. The other network predicts the source locations and other source features such as the source peak frequencies and amplitudes. To reduce the size of the input data to the network, we correlate the recorded traces with a central reference trace to allow the network to focus on the curvature of the input data near the zero-lag region. We train the networks to handle single, multi, and no event segments extracted from the data. Tests on a simple vertical varying model and a more realistic Otway field model demonstrate the approach's versatility and potential.
\end{abstract}




\section{INTRODUCTION}

Hydraulic fracturing is one of the most commonly used oil and gas extraction methods, especially in those reservoirs with dense rocks such as shale. Usually, water or other liquids are injected into the reservoir area to create high-pressure conditions, which eventually crack the dense rock to make the oil and gas flow more freely. The injection process often excites a massive number of micro-seismic events (Duncan, 2005), which can be monitored through sensors in monitoring well or on the earth's surface. Through the monitoring, the locations of the micro-seismic events are usually estimated, which in turn can help us learn the fracture development and monitor the fracturing process. Such information helps the engineers optimize their injection strategy (Warpinski, 2009). Hence, efficiently locating a vast number of micro-seismic events is vital to the hydraulic fracturing process.

During the monitoring of the fracturing, thousands of micro-seismic events are often recorded. Locating all these events can be a challenging and time-consuming task. Conventional ways to locate the sources include methods that are based on inverting traveltime picks of both P- and S-wave arrivals (Eisner et al., 2009). These methods are computationally fast, but they suffer from picking accuracy, as they require manual labor of picking. Often errors in picking lead to mislocation of micro-seismic sources. Manual picking of arrivals, however, can hardly be done accurately because the data usually has low signal-to-noise $(\mathrm{S} / \mathrm{N})$ ratio (Bose et al., 2009; Kummerow, 2010; Song et al., 2010).

Some other methods for locating micro-seismic events are based on the time-reversed imaging (TRI) technique, using the wavefields (Larmat et al., 2006, 2010; Lu et al., 2008; Steiner et al., 2008). Usually, the recorded data are back-propagated into a given model from their receiver locations. An imaging condition determines the source locations, such as the maximum energy imaging condition (Artman et al., 2010; Yang and Zhu, 2019), the geometric 
mean imaging condition (Nakata and Beroza, 2016) and the maximum variance imaging condition (Wang and Alkhalifah, 2017), to extract the source location. One advantage of TRI is that it does not require manual picking, especially when handling poor $\mathrm{S} / \mathrm{N}$ data. If a dense and uniform acquisition system is available, the wavefield will focus on the correct source locations. If a limited number of traces are available, TRI is not robust in accurately locating the sources. Many advanced strategies are proposed to mitigate the issue of irregular and sparse receivers (Fink et al., 2000; Tanter et al., 2000, 2001; Aubry et al., 2001; Jonsson et al., 2004; Larmat et al., 2010; Sava, 2011; Gallot et al., 2012).

Another category of micro-seismic imaging methods is based on inverting for better velocity models (Sun et al., 2016; Kaderli et al., 2018; Wang and Alkhalifah, 2018; Kamei and Lumley, 2014; Song et al., 2019a,b; Wang et al., 2020). The inversion is also responsible for velocity model building using a proper objective of data matching. Such data matching can also be used in image focusing (Witten and Shragge, 2017). However, migration-based and inversion-based methods require a high computational cost in simulating wavefields because tens of thousands of micro-seismic events may be recorded during the hydraulic fracturing processes.

With significant advances in computational resources, using machine learning algorithms to detect, locate, and interpret passive events automatically is gaining much attention. Human experts are responsible for preparing the training data, designing the algorithm's architecture, and finding the right set of hyper-parameters. Once the networks are well trained, they are purely automatic without human interactions. They do not require wavefield simulations, and thus, they vastly reduce the human and computational costs while maintaining high accuracy in locating events. Most studies in passive seismic data using machine learning methods are focused on micro-seismic event detection (Zheng et al., 2018; Zhang et al., 
2018; Huang et al., 2018). These methods aim to detect the micro-seismic events from the raw field monitoring data by training different neural networks using synthetic generated data. After detection, the micro-seismic events are inserted into conventional micro-seismic locating algorithms, which suffer from the challenges above. As a result, several techniques have already been proposed to locate earthquakes automatically. A convolutional neural network $(\mathrm{CNN})$ is trained using more than two thousand human-induced micro-seismic events recorded by two stations to classify the source locations roughly (Perol et al., 2018) into six regions. Kriegerowski et al. (2018) also trained a CNN using more than 2000 events from West Bohemia, recorded on nine local stations, to locate clustered earthquakes precisely. Recently, Zhang et al. (2020) use a fully convolutional network to predict the earthquakes in Oklahoma with only 30 stations. Mousavi and Beroza (2019) used a Bayesian-based deep learning algorithm to predict natural earthquakes. These papers are designed for global scale earthquakes with endurance for significant error.

In this study, we train two CNNs using correlated P-wave data as input. One network classifies the number of sources in the input data as a classification task. The other network predicts the source locations, the source wavelet peak frequencies, and the maximum amplitudes as a regression task. Once the CNNs are well trained, they can be used for realtime micro-seismic events locations on the raw monitored records. We first demonstrate the proposed method on a synthetic dataset generated with a simple $V(z)$ model. We add random noise to the data to show the noise sensitivity of the method. In real cases, we also face coherent noise, which may challenge the implementation. Adding random noise with sufficiently high energy is an excellent way to analyze the robustness of the approach, as it is often done. We then demonstrate the proposed method on the Otway model using a more realistic experiment setup to show the approach's effectiveness in more realistic situations. 
A moveout velocity analysis (MVA) version of the Otway model is used to generate the training dataset, and the true model is used in generating the testing set. We also add high levels of random noise to the datasets to show the robustness of the method. Wu et al. (2019) use synthetic data to train a neural network to identify faults in seismic images and use it in real data. We may further test the proposed method with field data in the next step while staying with this Otway model test in this paper.

Compared to what has been done in NN microseismic detection and location in the past, in this study: (1) We map raw recorded waveforms directly to the desired passive source parameters. (2) To do so practically, we use a correlation operation that helps us reduce long waveform segments to tiny windows of data. (3) We use a novel two neural network configuration, one for classification and the other for regression, to perform the mapping.

\section{METHOD}

We review deep convolutional neural networks as we relate them to our objective. We follow that by setting up the inputs and outputs of our network. Finally, we discuss the training process.

\section{Deep Convolutional Neural Network}

Machine learning (ML) is a branch of artificial intelligence (AI) that imitates the workings of the communication nodes in biological systems in processing data and creating patterns to use in decision making. An artificial neural network is a network or circuit of artificial neurons or nodes for solving AI problems (Hopfield, 1982). Artificial neurons are represented as weights in the matrix of a network. A positive weight models an excitatory connection 
between biological neurons, while negative values mean inhibitory connections. All inputs are modified by weight and summed. This activity is referred to as a linear combination. Finally, an activation function is applied to bring non-linearity to the model and control the output's amplitude. Deep learning is a subset of machine learning using multi-layers networks capable of supervised (or unsupervised) learning from labeled (or unlabeled) data. In recent years, with the development in computing resources, deep learning attracted more and more attention due to its better performance in solving ML problems. Deep learning uses multiple layers to extract higher-level features from the raw input progressively. For example, in image processing, shallower layers may identify edges, while deeper ones may identify the concepts relevant to a human, such as digits, letters, or faces (Deng and Yu, 2014).

The convolutional neural network is most commonly applied to analyzing visual imagery (LeCun et al., 1989; Hinton et al., 2012). The central concept behind deep CNNs is that the neural network connection is given by a convolution process in at least one of the layers (Goodfellow et al., 2016). The critical operation is given by a critical filter convolved with the input to capture the local features of the data corresponding to the filter size. The architecture of CNN is often driven by the application. We describe here the form we used in our application for both classification and regression. The output values of the convolutional layers are passed through an activation function to control the output value range and often down pooled by a max-pooling layer. The down-pooled data are fed to the next convolutional layer to extract more global features. After passing all the convolutional layers, the output values are often flattened to be fed to fully connected layers to generate the final predictions for the current epoch. Using the predictions, we can calculate a proper loss value between the predictions and the labels and then back-propagate the loss to train 
the network with a certain learning rate. The training process over sufficient epochs allows for good convergence, and the network will eventually learn the relation between the input data and their corresponding output labels. Then we can use the trained network to predict the labels of other similar featured data not involved in the training.

\section{The Neural Network Inputs And Outputs}

In micro-seismic experiments, the data recorded from the field are incredibly long, sometimes extending for days, weeks, or even months. There could be thousands of events hiding inside the record. Conventional pre-processing of the data includes splitting the data into several segments corresponding to the detected events. For many events, the pre-processing could consume much time and a high computational cost. We propose to process the raw data by cutting it into several equal segments along the time axis, preferably containing three events or fewer for this study. When handling real data that we expect to have more events, we may further train the network with training data that contains more events. We here focus on showing the potential of this method. We pick a reference trace from every segment and correlate it with all the traces in the segment using:

$$
D(x, \tau)=d_{r e f}(t) * d(x, t)
$$

where $d(x, t)$ is the data segment, $d_{r e f}$ is the chosen reference trace from the data segment, * is the correlation operation and $\tau$ is the lag of the correlation operation. The data before and after the correlation are shown in Figure 1. Note that the single event in this segment is set to approximately zero lag after the correlation operation. Thus, we can represent the data segment with a smaller time window around the zero time lag regardless of the number of events in the segment. In this way, we may reduce the CNN input data size. 
Only cross-correlation cross-talk will have large time lags. Although, in this case, the events that might be separated in time may overlap in the cross-correlation, we still can train the network to learn to identify each of them. In other words, we may significantly reduce the raw record from several hours long to less than one second, which is a significant saving. In such a case, there might be more events recorded and correlated in the same input file. Thus, we may need to train the neural network with training data containing more than one event before using it. Besides, we treat all the data segments the same way without detecting the events, which reduces manual interaction. Note that the whole pre-processing is automated. We use such correlated data with a short time lag window as the input data to the network.

The output labels of the network contain all the corresponding source information, including the event locations in 2D $(X \& Z)$, the source wavelet peak frequencies $(F)$, the amplitudes $(A)$, and the number of the sources in the segment $(N)$. We choose to train the network by predicting such output labels because they have their signatures in the input data. The location $X$ coordinate information is driven by the event apex horizontal position in the recorded data; $Z$ is defined mainly by the moveout (the depth of the source influences the curvature of the events ); $F$ is related to the wavelength or the thickness of the event; $A$ is defined by the maximum energy recorded. Once the labels have their unique signatures in the data, one can add more output labels to the network training.

\section{The Convolutional Neural Networks Architectures And Training}

In this study, we use the two networks, shown in Figures 2 and 3. One network predicts the number of events $N$ in the input data as a classification problem. The other network predicts the source locations $X$ and $Z$, the peak frequencies $F$, and the maximum ampli- 
tudes of the events $A$ as a regression problem. We decided to confine the number of events within a segment to three as a maximum in the following examples. However, increasing that number is straightforward and has no bearing on the framework.

Figure 2 shows the classification CNN that classifies the input data to the correct source number classes. Four convolutional layers and one global average pooling layer all pass through a tanh activation function. Recently some popular fully convolutional neural networks have been proposed to avoid using fully connected layers to minimize the number of parameters while maintaining high performance. To achieve this, Lin et al. (2013) use global average pooling (GAP), which acts as a structural regularizer, preventing over-fitting during training. Zhou et al. (2016) suggest that GAP improves the performance of classification while reducing the number of neurons to save training time. Thus, we use GAP when building the classification CNN for this study. Instead of using down-pooling layers, we set the stride value of each convolutional layer to two, which down pools the input tensor by half in both axes after each convolutional layer. 10,20,40, 80 convolutional filters are set to the first, the second, the third, and the last convolutional layer, respectively. We use the tanh activation function between layers because seismic data have both positive and negative values.

The loss value of the classification network is calculated by the cross-entropy function between the predicted and true labels probability distributions:

$$
H(T, P)=-E_{T}[\log P]
$$

where $\mathrm{P}$ and $\mathrm{T}$ refer to predicted and true probability distributions, respectively. $E_{P}$ is the expected value operator with respect to the distribution $\mathrm{P}$. This cross-entropy loss function provides a probability distribution of each possible output label. We back-propagate the loss to train the network and optimize the predicted distribution that describes the true 
one by a stochastic gradient descent (SGD) optimizer. Other popular optimizers such as the Adam (Kingma and Ba, 2014) optimizer can also be used in the algorithm. From our experience, SGD shows reasonably good convergence for this task, and thus, we use this optimizer in our algorithm.

Figure 3 shows the regression $\mathrm{CNN}$ architecture that predicts the source locations $(X$ and $Z$ ), the peak frequencies $(F)$, and the maximum amplitudes $(A)$. The mean squared error (MSE) loss for the regression network is calculated by:

$$
L=\sum_{i=1}^{n}\left(\left|X_{P}^{i}-X_{T}^{i}\right|^{2}+\left|Z_{P}^{i}-Z_{T}^{i}\right|^{2}+\left|F_{P}^{i}-F_{T}^{i}\right|^{2}+\left|A_{P}^{i}-A_{T}^{i}\right|^{2}\right),
$$

where $i$ is the source number, and $n$ is the maximum number of sources assumed. This loss function is back-propagated to train the neural network to minimize the loss function. We still use an SGD method to optimize the parameters in the network. The output labels are all normalized to $[0,1]$ or $[-1,1]$ as it helps balance the training for the various labels. For example, the source wavelet peak frequencies in our following experiments are randomly generated from $5-15 \mathrm{~Hz}$. Thus, we first subtract all the frequency labels by $5 \mathrm{~Hz}$ and then divide the labels by the new maximum value of 10 to normalize the frequency labels to the range of $[0,1]$. A similar normalization is applied to other labels. There are six convolutional layers and three fully connected layers. The activation function remains the same as the first network. A max-pooling layer with a $2 * 2$ pooling size is used to down-scale the data by half in each axis between the layers.

Both the training and testing datasets are correlated recorded waveforms calculated using equation 1. Figure 4a-4d show example training data with single, double, triple, and zero events, respectively. The inputs have distinct features (such as apex locations, moveouts) for the network to learn. The network setup in this study is expecting three events per training example $(n=3)$. To train for single or double events, we assume that such events 
include coinciding sources. We replicate the labels to all three expected event labels for a single event as if we had three sources with the same labels. We replicate one of the two events to fill in the three events requirements for the two events. For example, single event data have the frequency label $[0.5,0.5,0.5]$ after the normalization. Double event data have the frequency label $[0.5,0.5,0.8]$, and triple events data have the label $[0.5,0.3,0.8]$, if all three events have different frequencies. In other words, we assume the single event to be a triple event of the same frequency. This process implies that we assume there are $n$ events in all non-zero events (based on classification) input data. However, there could be two or three sources excited under precisely the same condition; thus, the events are entirely overlapped.

To help the network learn well, we keep the order of arrival time consistent in the labels, so earlier arrival events are in the first of the three-event labels and the latter in the last. As mentioned earlier, this ordering will not matter for a single event segment, and the labels are for the same event. We also normalize the labels to range between $[0,1]$ or $[-1,1]$ to stabilize the training.

Thus, the network is used to learn the nonlinear relationships between the input data and the output labels. Increasing the number of convolutional layers or the number of neurons in each layer may improve the performance of some training sets. However, too many neurons in the network may lead to over-fitting, which means the network memorizes the data instead of learning them. Thus, the network, in this case, performs poorly on test data. Therefore, the choice of network parameters is quite crucial for optimal performance. One way to evaluate the over-fitting problem is to include a validation set in the training process by separating a small part of the training set for validation and not involving it in the back-propagation (training). The validation set is used to monitor the performance of 
data not used in training. If the validation set loss does not decrease or even increases while the training loss drops, it means we are probably facing the over-fitting issue.

The whole workflow can be described as (1) Train the classification network with all the training data; (2) Train the regression network with all the non-zero events training data; (3) Test the classification network with a test set and filter out the non-zero events data; (4) Test the regression network with the non-zero events testing data in step (3).

The proposed approach has advantages over current networks in many ways. It does not require event detection, nor it requires manual picking. We treat the raw record by first slicing the raw recorded into several segments, usually of equal size for simplicity. Each segment could be one hour long, as an example. Then, we pick a reference trace and correlate it with the data. In this way, we convert the one-hour-long raw record into seconds or even less than a second long, and we input such reduced data into the network. Compared to using raw records as input data, the input data size is reduced, and thus, the training and testing time is practical. We input the correlated data into the two networks. We obtain information on the number of events to help us understand the outputs from the other network and source locations and other parameters of the micro-seismic event.

The framework for applying this approach to real data may include additional training to adapt the NN model to real data. We propose first training the network on synthetic data using the best available velocity model extracted from real or other sources. The synthetic data should have the same acquisition setup as the real data. Further training on some previously inverted labels from the data can help improve the network performance (Kriegerowski et al., 2018). We intend to show such an application in a follow-up paper. In this paper, we focus on the concept and synthetic tests. 


\section{EXAMPLES}

We first test this approach on a simple vertically varying model focusing on the ability of the network to predict the events and their characteristics. We then apply the approach on a more realistic model in which we use a smoothed version of the velocity model (like those that comes from imaging) to generate data for training the network and then use the true model to generate data for testing the network (as such data should represent the true model). We perform the second experiment with micro-seismic events placed at the reservoir area to represent more realistic conditions.

\section{Synthetic Test On A $V(z)$ Model}

The training and testing datasets for the experiment are generated synthetically. We compute waveform records corresponding to sources distributed randomly through the whole velocity model to create the data. In our experiment, the velocity is linearly increasing with depth $(V(z)$ model), ranging from $2 \mathrm{~km} / \mathrm{s}$ to $2.64 \mathrm{~km} / \mathrm{s}$. The model size is $0.64 \mathrm{~km}$ by $0.64 \mathrm{~km}$ with a $10 \mathrm{~m}$ spatial spacing. The monitoring system includes sensors at every grid point on the earth's surface (total of 64 receivers). The source parameters are randomly generated, which means the source positions $(X, Z=[0.05,0.63] \mathrm{km})$, the peak frequencies $(F=[5-15] \mathrm{Hz})$, and the maximum amplitudes $(A=[0.5-1])$ are all random numbers within the ranges. When generating the data, up to three sources $(N=[0-3])$ with random values are used as the sources so that the maximum source number is three in the experiment, and in some cases, we might have no sources. For each segment, we choose the central trace as a reference trace and apply the correlation operation mentioned in equation 1 . We limit the correlated data to a $0.4 \mathrm{~s}$ time lag window and normalize them 
by the maximum value of all the data segments to form the input data to the network. So in line with our objective, we pre-process the data with the correlation step, eliminating the source origin time information as all the events will be pulled to the zero time-lag position. Thus, we mainly focus only on event locations, frequencies, and magnitudes. In total, 5332 training data and their labels are prepared, including 1333 samples each for the zero, single, double and triple events cases.

The input data examples are displayed in Figure 4a-4d. Random noise of $50 \%$ level with respect to the maximum amplitude has been added to the data. We set the training batch size to 100 throughout the experiment.

The classification network error rates for the training and validation datasets are shown in Figure 5a. The error rates decrease to less than 3\%. The regression network training and validation loss curves are shown in Figure 5b. The smooth decrease is mainly due to the learning rate schedule strategy. The initial learning rate (0.01) decreases $50 \%$ every 1000 epochs. The loss function may decrease further, but we choose to stop the training at 5000 epochs. The fact that the loss function did not converge to zero is one sign that we are not over-fitting. Luckily, the neural network training is not sensitive to random noise in the inputs. In fact, in some situations, the random noise may help the training as we add some values to all zero inputs, and this step often prevents instability in training.

To better analyze the predicted results of the trained networks, we generate separate test sets with sources on a regular grid to test the network. Specifically, we generate 1332 test data with random parameters that include 333 samples for each event case to test the classification performance of the network. The predicted largest probability of the source numbers $N$ in the data are shown as the red line in Figure 6 and the corresponding true source numbers are shown as the blue line. The accuracy of the test set reaches $97.75 \%$, 
which is generally good. For most single and zero source cases, the accuracy is almost $100 \%$. Only a few errors in multi-sources cases occur, which makes sense because some nearby sources may appear like a single source.

We generate 225 test data for the single-source case in which sources are excited on regular grid points, which has 15 rows in the $Z$ direction and 15 columns in the $X$ direction, to better demonstrate the predicted source location accuracy with respect to the spatial positions. The sources are excited with random $F$ and $A$. Then we randomly select two (or three) single event data and sum the selected data to generate the multi-sources cases test set.

Figure 7a-7f show the predicted source locations compared to the true source locations and their corresponding error maps for single, double, and triple source cases. The predicted and the true source locations are marked by the red and blue dots, respectively. We can generally see that the predicted sources are quite close to the true source locations and many predicted sources even overlap their true locations. The error maps also describe how well the network predicts the labels in different areas. They are generated by calculating the differences between the true and predicted source locations on regular grid points in the source region and interpolating the values to a denser grid for better visualization. For multi-events predictions, the errors are calculated by averaging all the sources' absolute distance errors in the same input data. In general, the middle parts of the model show smaller errors than at the edges. It is mainly because the middle parts of the model are better illuminated than the edges. The errors for the different scenarios range from a few meters to $50 \mathrm{~m}$ maximum. In general, the network seems to do well in locating micro-seismic events.

To test the $F$ accuracy, we generate 100 data examples with fixed source locations for 
each parameter. The five sources are located uniformly from the top-left corner to the bottom-right corner. The frequencies are increased by $0.1 \mathrm{~Hz}$ from 5 to $15 \mathrm{~Hz}$ to analyze the $F$ prediction performance for frequency. The same frequencies are used to generate the multi-sources data. Thus, we can analyze the network performance as a function of frequency. The curves for the predicted and the true source peak frequencies $F$ are shown in Figure 8a-8c for the single, double, and triple events cases, respectively. The errors of the predicted labels are averaged over all the absolute error values of the same frequency for the five sources. The predicted (red) and the true (blue) curves for the single event cases match each other. They almost overlap since the errors between the two curves never exceed 0.3 $\mathrm{Hz}$, except for only very few sources. The multi-events cases frequencies are generally well predicted with the maximum error lower than $1 \mathrm{~Hz}$. However, the accuracy is slightly lower than in the single-event case. In multi-sources cases, events may overlap and change the thickness of the events, which will confuse the network. Generally, the lower frequencies are slightly better predicted than the higher frequencies, which is probably expected. It is mainly due to those with higher frequencies having smaller wavelengths (less energy) in the input images and, thus, dominated by the more energetic lower frequency events.

We repeat the same analysis to $A$ that the amplitudes increase by 0.005 from 0.5 to 1 . The amplitude predictions and the corresponding true values are shown in Figure 9a-9c for the single, double, and triple events cases, respectively. The predicted value absolute errors are also averaged over all the labels with the same amplitude of the five sources. Similar to the frequency results, the single event cases have the best predictions. For multi-events cases, the predictions are worse due to the complexity of the waveforms. The amplitudes are slightly less accurate than the frequencies, mostly due to the normalization of the input data, which we have mentioned, to maintain the stability of the training. 
We may obtain better results for the frequencies and the amplitudes predictions by adding more training data. In general, more training data in machine learning leads to better results, but it will also increase the cost of the training. The balance between the training cost and the performance is also an issue which needs further attention. However, the benefits of training well (even if costly) such a network as it can be used for long monitoring periods might outweigh the cost.

\section{A More Realistic Experiment: The Otway Model}

The Otway model is extracted from a $\mathrm{CO}_{2}$ injection field near Melbourne, Australia. The model should reflect the elasticity of the subsurface there. We keep the CNNs the same as for the $V(z)$ model example. The velocity model has a size of $2.442 \mathrm{~km}$ by $1.968 \mathrm{~km}$ with a $6 \mathrm{~m}$ spatial interval in the $X$ and $Z$ directions. We smooth the model using a triangular smoothing operator with a window size of 15 by 10 samples in the $\mathrm{X}$ and $\mathrm{Z}$ directions, respectively. Hence, it reflects the resolution we may get from migration velocity analysis (MVA). We use the smoothed velocity model, which is shown in Figure 10a, to generate the training set and use the true model, which is shown in Figure 10b, to generate the testing set, representing the real earth. The sources are randomly ignited within the blue box area, extending from 0.75 to $1.75 \mathrm{~km}$ in the $X$ direction and $1.4-1.64 \mathrm{~km}$ in the $Z$ direction. This location is assumed to be where the $\mathrm{CO}_{2}$ is injected into the field. Here we assume the source area reflects a horizontal well injection process commonly used in shale gas exploration. We situate 100 receivers on the surface of the model with a $24 \mathrm{~m}$ interval. Similarly, the other source parameters are also randomly generated, within the same ranges used for the $V(z)$ model test. All the labels are normalized to the range of $[0,1]$. A total of 10, 000 data examples have been generated as the training set - - 2500 data for each event 
number case $(N=[0,3])$.

The non-zero event data examples for the training and testing sets can be found in Figure 11a and 11b, respectively. Random noise of a $50 \%$ level with respect to the maximum amplitude was added to the data. The batch size is 100 in the experiment.

The classification training and validation accuracy curves are shown in Figure 12a. We use an SGD optimizer. The learning rate starts at 0.1 and decreases by $50 \%$ every 100 epochs. The final error rates on both the training and validation sets are less than $1 \%$. A large amount of training data helps speed up the convergence of the loss function with respect to the epoch number, while for each epoch, the training time increases. Similar to the $V(z)$ model experiment, to better understand the performance of the trained networks, we generate several test sets for different parameter testing. 2000 data samples have been generated to test the classification network - - 500 data samples for each source number case. The testing set classification result is shown in Figure 13. The accuracy reaches $96.1 \%$ (78 tests with errors out of 2000). Recall, the training set and the testing set are generated from different velocities. Under such a condition, the testing accuracy is high. The single and zero source cases are well predicted. However, the multi-sources cases are less accurately predicted due to the complexity of their waveforms, which can easily confuse the neural network.

The loss curves of the regression training and validation sets are shown in Figure 12b. In the first 100 epochs, the loss value drops rapidly, and then the loss decreases smoothly until the end. The rapid drop, in the beginning, is mainly due to the network quickly learning to produce an acceptable average value of the output labels, which is related to fitting. After that, the network learns more about the input data features and gives better predictions. We generate 600 data samples in which the sources were excited on a regular grid in the 
source region, which is shown as the blue dots in Figure 10b, to better demonstrate the predicted source location accuracy with respect to the spatial position of the source. 100 sources are ignited with random $F$ and $A$ to generate the single event test set. Then, we randomly select two (or three) single-event data and add the selected data to form the multi-sources cases test set. In total, 100 single event data, 200 double events data, and 300 triple events data have been generated to test the source location prediction accuracy regarding the spatial distribution. The predicted source locations of the regular-grid testing set and their corresponding error maps are shown in Figure 14a-14f. The true and predicted source locations are marked as the blue and red dots, respectively. The true source locations are surrounded by the well-predicted source locations. The maximum error is less than 50 $\mathrm{m}$, and the average error is only about $15 \mathrm{~m}$. As expected, the larger errors occur on the edges, and the smaller ones are in the region's middle parts, which is realized earlier in the $V(z)$ model experiment.

We use five source locations, which are shown in yellow in Figure 10b, to generate the test data for the frequency prediction. We use the same approach in generating the test data as we did in the $V(z)$ model test set. The curves of the predicted and true $F$ for the different cases, and their corresponding absolute errors, are shown in Figure 15a-15c. For all cases, the predicted (red) curves and the true (blue) curves match each other and almost overlap. The multi-events frequencies are better predicted than the $V(z)$ model test results, which we believe is mainly due to the larger training dataset. Remember that we have almost double the training examples for the Otway model test. The lower frequencies are slightly better predicted than the higher frequencies, as the higher frequencies are more likely to be influenced by the other events and noise. The fewer events the input data contains, the better the network performs. It makes sense that the more events we have in the data, the 
more complex the data becomes, which makes it harder for the network to identify each event.

Same sets are generated to test the amplitude accuracy as the $V(z)$ model test set. The sources are kept in the same location given in yellow in Figure 10b. The amplitude predictions and the corresponding true values are shown in Figure 16a-16c. Similarly, the prediction errors are averaged over all the absolute values of the errors that have the same $A$. Unfortunately, the amplitudes are not as well predicted as in the $V(z)$ model test results, although we have more training data. The average error is about 0.1 out of 0.5 , which is reasonably low. We believe this may be caused by the even more complex waveforms generated from the Otway model. After the normalization of the amplitudes, the input data could be too biased for the network to learn the amplitude regularity. We also find that the larger $A$ values are better predicted than the lower ones, which is expected in data with noise. The data with larger source energy have larger weights in training the network, while the lower amplitude data will have a smaller influence, which agrees with our analysis. The weaker events have a smaller signal-to-noise $(\mathrm{S} / \mathrm{N})$ ratio, while the stronger ones have a greater $\mathrm{S} / \mathrm{N}$ ratio. We may increase the accuracy of the amplitude prediction by training the network to suppress the noise and unnecessary events, and this aspect is worth studying in the future.

\section{Comparison With The Traditional Time-reversal Imaging}

In this section, we compare the ML locating accuracy with traditional time-reversal imaging (TRI) results better to demonstrate the advantages of the proposed ML method.

We use the most common TRI locating method, based on the maximum energy imaging condition (Artman et al., 2010) under the same experiment set up in the Otway model 
test. The testing data generated by the true Otway velocity model is reversed in time and injected as sources at the corresponding receiver locations using the smoothed velocity model. Then a maximum energy imaging condition is applied, which provides the maximum energy position in the whole back-propagated wavefield volume. Such maximum energy positions are thus referred to as the migrated source locations.

Figure 17a-17b show the histograms of the located events accuracy of the machine learning and the maximum energy TRI methods, respectively. We find that the average errors of the two methods are almost at the same level, that the machine learning method provides an average error of $20 \mathrm{~m}$ and the TRI method provides an average error of $15 \mathrm{~m}$. Considering that the velocity is accurate, the TRI method is robust because it is the adjoint of modeling. Thus, the errors in the location do not exceed $25 \mathrm{~m}$. On the other hand, for the ML method, about ten events had errors exceeding $30 \mathrm{~m}$, which affected the average. However, the ML approach is far more efficient (instant), which is an important feature for real-time monitoring. On a single workstation, the total time to predict all 200 test examples is two minutes. Meanwhile, it takes more than a minute to perform the propagation for a single source TRI application. The difference in cost will increase dramatically for realistic 3D cases, which is the real objective of this development. TRI also requires that we detect and isolate events to achieve the described efficiency and accuracy. If we reverse a one-hour data segment in time, the cost will be far higher. Not to mention that if we had more than one event in the segment, the maximum energy imaging condition could only capture the strongest event.

21 


\section{DISCUSSIONS}

The training process is not only accurate but also efficient. The training process on a single GPU (Nvidia Titan RTX) took only six hours, and the well-trained network can predict all the testing set labels in a few minutes. This feature will be attractive when we use the network to monitor a field, especially when comparing it with conventional methods requiring manual picking or solving partial differential equations. Thus, we propose an automated method with good accuracy, especially in locating the micro-seismic sources, while saving computational cost and manual efforts. Also, in the field monitoring process, even if the velocity model is updated using the new data, the network's training considering the new velocity can be cheap through transfer learning. One form is given by using the current neural network model as the initial model for the new training. For this ML implementation, we focus on the micro-seismic event location and magnitude and the peak frequency of the source signature. However, we cannot identify the origin time of the event within the cross-correlated segment using this approach. Though the origin time is important in earthquake seismology, it tends to be less important for reservoir monitoring and fracture mapping. So to allow our method to map data directly with a reasonable size input to the network and less variance in the input dataset, we opted to sacrifice the origin time information using a correlation step. The passive event location accuracy is dependent on station locations, velocity model, and the physics involved in the training. In this paper, we assume that a good enough MVA based model is provided, and the receiver locations are known. We focused on the acoustic assumption, but the same approach can be used for elastic medium. If the monitoring system or the velocity model changes, we may need to retrain the network (i.e., transfer learning) with new data (corresponding to the new velocity and acquisition) to adapt to the new conditions. In the paper, we demonstrate the method 
with up to three events in a single segment, and we claim that no limitation will prevent us from adding more events if needed. Because we assume more events in one segment, the more complex the data is. Thus, to perform better on such complex multi-event data, the network may need more training data to adapt to the complexity. Moreover, the transition from synthetic training to field applications is often the most important and challenging part of machine learning based work, and a large drop in performance may be observed (Tschannen et al., 2020). Thus, implementing the proposed method to field data will be challenging and require more study. In this paper, we focus on showing a proof of concept, and the application to field data requires further study, which is beyond the scope of the paper.

\section{CONCLUSION}

We developed a CNN framework that can efficiently and accurately predict the microseismic source locations, peak frequencies, and maximum amplitudes from micro-seismic monitoring data. To prepare the data and avoid event identification or picking, we crosscorrelate a central trace from a data segment with the rest of the traces. This process moves all the events in the segment closer to the zero-lag area offering a more compact data representation. We input such data into a classification network trained to determine how many events in the data segment. We input the data into another trained network to extract the micro-seismic event features if events are present. We train the network on correlated acoustic waveforms from a $V(z)$ model and the Otway model to test this framework. We trained the network on data simulated using a smooth version of the true velocity model for the Otway example to replicate what we would get from tomography methods and then test the network on data corresponding to the true model. From our results, a source with 
low frequency, high energy, and located in a better-illuminated part of the model is easier to predict. On the other hand, a source with high frequency, low energy, and located on the model edges is harder to predict accurately. In addition, the network is generally insensitive to random noise, which makes it more stable and useful when dealing with real data, though field data tests represent an important milestone that has yet to be reached for the method to be validated.

\section{ACKNOWLEDGMENTS}

We thank KAUST for supporting this research. We also thank the team of SWAG for their help during the research. Special thanks to KAUST Supercomputing Laboratory for providing the computing resource. 


\section{REFERENCES}

Artman, B., I. Podladtchikov, and B. Witten, 2010, Source location using time-reverse imaging: Geophysical Prospecting, 58(5), 861-873.

Aubry, J.-F., M. Tanter, J. Gerber, J.-L. Thomas, and M. Fink, 2001, Optimal focusing by spatio-temporal inverse filter. ii. experiments. application to focusing through absorbing and reverberating media: The Journal of the Acoustical Society of America, 110(1), $48-58$.

Bose, S., H.-P. Valero, Q. Liu, R. Shenoy, and A. Ounadjela, 2009, An automatic procedure to detect microseismic events embedded in high noise, in SEG Technical Program Expanded Abstracts 2009: Society of Exploration Geophysicists, 1537-1541.

Deng, L., and D. Yu, 2014, Deep learning: methods and applications: Foundations and trends in signal processing, 7(3-4), 197-387.

Duncan, P. M., 2005, Is there a future for passive seismic?: First Break, 23(6), 111-115.

Eisner, L., P. M. Duncan, W. M. Heigl, and W. R. Keller, 2009, Uncertainties in passive seismic monitoring: The Leading Edge, 28(6), 648-655.

Fink, M., D. Cassereau, A. Derode, C. Prada, P. Roux, M. Tanter, J.-L. Thomas, and F. Wu, 2000, Time-reversed acoustics: Reports on progress in Physics, 63(12), 1933.

Gallot, T., S. Catheline, P. Roux, and M. Campillo, 2012, A passive inverse filter for green's function retrieval: The Journal of the Acoustical Society of America, 131(1), EL21-EL27.

Goodfellow, I., Y. Bengio, and A. Courville, 2016, Deep learning: MIT Press.

Hinton, G. E., N. Srivastava, A. Krizhevsky, I. Sutskever, and R. R. Salakhutdinov, 2012, Improving neural networks by preventing co-adaptation of feature detectors: arXiv preprint arXiv:1207.0580.

Hopfield, J. J., 1982, Neural networks and physical systems with emergent collective com- 
putational abilities: Proceedings of the national academy of sciences, 79(8), 2554-2558.

Huang, L., J. Li, H. Hao, and X. Li, 2018, Micro-seismic event detection and location in underground mines by using convolutional neural networks (cnn) and deep learning: Tunnelling and Underground Space Technology, 81, 265-276.

Jonsson, B. L. G., M. V. de Hoop, M. Gustafsson, and V. H. Weston, 2004, Retrofocusing of acoustic wave fields by iterated time reversal: SIAM Journal on Applied Mathematics, 64(6), 1954-1986.

Kaderli, J., M. D. McChesney, and S. E. Minkoff, 2018, A self-adjoint velocity-stress full-waveform inversion approach to microseismic source estimation: Geophysics, 83(5), R413-R427.

Kamei, R., and D. Lumley, 2014, Passive seismic imaging and velocity inversion using full wavefield methods, in SEG Technical Program Expanded Abstracts 2014: Society of Exploration Geophysicists, 2273-2277.

Kingma, D. P., and J. Ba, 2014, Adam: A method for stochastic optimization: arXiv preprint arXiv:1412.6980.

Kriegerowski, M., G. M. Petersen, H. Vasyura-Bathke, and M. Ohrnberger, 2018, A Deep Convolutional Neural Network for Localization of Clustered Earthquakes Based on Multistation Full Waveforms: Seismological Research Letters, 90(2A), 510-516.

Kummerow, J., 2010, Using the value of the crosscorrelation coefficient to locate microseismic events: Geophysics, 75(4), MA47-MA52.

Larmat, C., J.-P. Montagner, M. Fink, Y. Capdeville, A. Tourin, and E. Clévédé, 2006, Time-reversal imaging of seismic sources and application to the great Sumatra earthquake: Geophysical Research Letters, 33(19), L19312.

Larmat, C. S., R. A. Guyer, and P. A. Johnson, 2010, Time-reversal methods in geophysics: 
Physics Today, 63(8), 31-35.

LeCun, Y., B. Boser, J. S. Denker, D. Henderson, R. E. Howard, W. Hubbard, and L. D. Jackel, 1989, Backpropagation applied to handwritten zip code recognition: Neural computation, 1(4), 541-551.

Lin, M., Q. Chen, and S. Yan, 2013, Network in network: arXiv preprint arXiv:1312.4400.

Lu, R., M. N. Toksöz, and M. E. Willis, 2008, Locating microseismic events with time reversed acoustics: a synthetic case study, in SEG Technical Program Expanded Abstracts 2008: Society of Exploration Geophysicists, 1342-1346.

Mousavi, S. M., and G. C. Beroza, 2019, Bayesian-deep-learning estimation of earthquake location from single-station observations: arXiv preprint arXiv:1912.01144.

Nakata, N., and G. C. Beroza, 2016, Reverse time migration for microseismic sources using the geometric mean as an imaging condition: Geophysics, 81(2), KS51-KS60.

Perol, T., M. Gharbi, and M. Denolle, 2018, Convolutional neural network for earthquake detection and location: Science Advances, 4(2), e1700578.

Sava, P., 2011, Micro-earthquake monitoring with sparsely sampled data: Journal of Petroleum Exploration and Production Technology, 1(1), 43-49.

Song, C., T. Alkhalifah, and Z. Wu, 2019a, Microseismic event estimation and velocity analysis based on a source-focusing function: Geophysics, 84(3), KS85-KS94.

Song, C., Z. Wu, and T. Alkhalifah, 2019b, Passive seismic event estimation using multiscattering waveform inversion: Geophysics, 84(3), KS59-KS69.

Song, F., H. S. Kuleli, M. N. Toksöz, E. Ay, and H. Zhang, 2010, An improved method for hydrofracture-induced microseismic event detection and phase picking: Geophysics, 75(6), A47-A52.

Steiner, B., E. H. Saenger, and S. M. Schmalholz, 2008, Time reverse modeling of low- 
frequency microtremors: Application to hydrocarbon reservoir localization: Geophysical Research Letters, 35(3), L03307.

Sun, J., Z. Xue, T. Zhu, S. Fomel, and N. Nakata, 2016, Full-waveform inversion of passive seismic data for sources and velocities, in SEG Technical Program Expanded Abstracts 2016: Society of Exploration Geophysicists, 1405-1410.

Tanter, M., J.-F. Aubry, J. Gerber, J.-L. Thomas, and M. Fink, 2001, Optimal focusing by spatio-temporal inverse filter. i. basic principles: The Journal of the Acoustical Society of America, 110(1), 37-47.

Tanter, M., J.-L. Thomas, and M. Fink, 2000, Time reversal and the inverse filter: The Journal of the Acoustical Society of America, 108(1), 223-234.

Tschannen, V., N. Ettrich, M. Delescluse, and J. Keuper, 2020, Detection of point scatterers using diffraction imaging and deep learning: Geophysical Prospecting, 68(3), 830-844.

Wang, H., and T. Alkhalifah, 2017, Time reversal migration for passive sources using a maximum variance imaging condition: Presented at the 79th EAGE Conference and Exhibition 2017.

— 2018 , Microseismic imaging using a source function independent full waveform inversion method: Geophysical Journal International, 214(1), 46-57.

Wang, H., Q. Guo, T. Alkhalifah, and Z. Wu, 2020, Regularized elastic passive equivalent source inversion with full-waveform inversion: Application to a field monitoring microseismic data set: Geophysics, 85(6), KS207-KS219.

Warpinski, N., 2009, Microseismic monitoring: Inside and out: Journal of Petroleum Technology, 61(11), 80-85.

Witten, B., and J. Shragge, 2017, Microseismic image-domain velocity inversion: Marcellus shale case studymicroseismic inversion: Geophysics, 82(6), KS99-KS112. 
Wu, X., L. Liang, Y. Shi, and S. Fomel, 2019, Faultseg3d: Using synthetic data sets to train an end-to-end convolutional neural network for 3d seismic fault segmentation: GEOPHYSICS, 84(3), IM35-IM45.

Yang, J., and H. Zhu, 2019, Locating and monitoring microseismicity, hydraulic fracture and earthquake rupture using elastic time-reversal imaging: Geophysical Journal International, 216(1), 726-744.

Zhang, X., C. Yuan, J. Zhang, S. Liu, Z. Chen, and W. Li, 2018, Automatic microseismic detection and location via the deep-convolutional neural network, in SEG Technical Program Expanded Abstracts 2018: Society of Exploration Geophysicists, 3057-3061.

Zhang, X., J. Zhang, C. Yuan, S. Liu, Z. Chen, and W. Li, 2020, Locating induced earthquakes with a network of seismic stations in Oklahoma via a deep learning method: Scientific reports, 10(1), 1-12.

Zheng, J., J. Lu, S. Peng, and T. Jiang, 2018, An automatic microseismic or acoustic emission arrival identification scheme with deep recurrent neural networks: Geophysical Journal International, 212(2), 1389-1397.

Zhou, B., A. Khosla, A. Lapedriza, A. Oliva, and A. Torralba, 2016, Learning deep features for discriminative localization: Proceedings of the IEEE conference on computer vision and pattern recognition, 2921-2929. 


\section{LIST OF FIGURES}

1 Data before and after the correlation operation.

2 CNN with a global average pooling layer for solving classification issues. The yellow blocks refer to convolutional operators. The numbers on the top refer to the operator sizes and the data sizes. The numbers of each convolutional layer size indicate the output channels * output data size in the first dimension * output data size in the second dimension.

3 CNN with fully connected layers for solving regression issues. The yellow blocks refer to convolutional operators. The numbers on the top refer to the operator sizes and the data sizes that indicate the output channels * output data size in the first dimension * output data size in the second dimension.

4 Example correlated data with (a) single (b) double (c) triple (d) zero events. Random noise of $50 \%$ level has been added.

5 (a) Training (blue) and validation/testing (green) error rate curves for classification; (b) Training (blue) and validation/testing (green) loss curves for regression.

6 The predicted (red) and the true source numbers $N$ in each testing data.

$7 \quad V(z)$ model (a) single (c) double, and (e) triple sources cases coordinates $X \& Z$ predictions. Corresponding error maps for (b) single (d) double, and (f) triple sources cases. The yellow color indicates large errors in space, and the blue color indicates small errors. The red dots indicate all the event locations in the multi-events data.

8 Predicted source frequencies $F$ for (a) single (b) double (c) triple sources cases. 100 frequencies are shown for each case. True and predicted labels are shown as the blue and red curves, respectively.

$9 \quad$ Predicted source amplitudes $A$ for (a) single (b) double (c) triple sources cases. 
100 examples are shown for each case. True and predicted labels are shown as the blue and red curves, respectively.

10 (a) Smoothed (MVA) Otway P-wave velocity model used for generating the training set. (b) True model used for generating the testing set on regular grids.

11 Non-zero example correlated data in (a) training (b) testing set with (left) one (middle) two (right) three sources. Random noise of $50 \%$ level has been added.

12 (a) Classification and (b) regression training and validation loss curves.

13 The predicted (red) and the true source numbers $N$ in each testing data on the Otway model.

14 Otway model (a) single (c) double, and (e) triple sources cases coordinates $X \& Z$ predictions. Corresponding error maps for (b) single (d) double, and (f) triple sources cases. The yellow color indicates larger errors in space and the blue color indicates smaller errors. The red dots indicate all the event locations in the multi events data.

15 Predicted source frequencies $F$ for (a) single (b) double (c) triple sources cases. 100 frequencies are shown for each case. True and predicted labels are shown as the blue and red curves, respectively.

16 Predicted source amplitudes $A$ for (a) single (b) double (c) triple sources cases. 100 amplitudes are shown for each case. True and predicted labels are shown as the blue and red curves, respectively.

17 (a) Machine learning locating accuracy histogram verses. (b) traditional timereversal imaging accuracy histogram. 


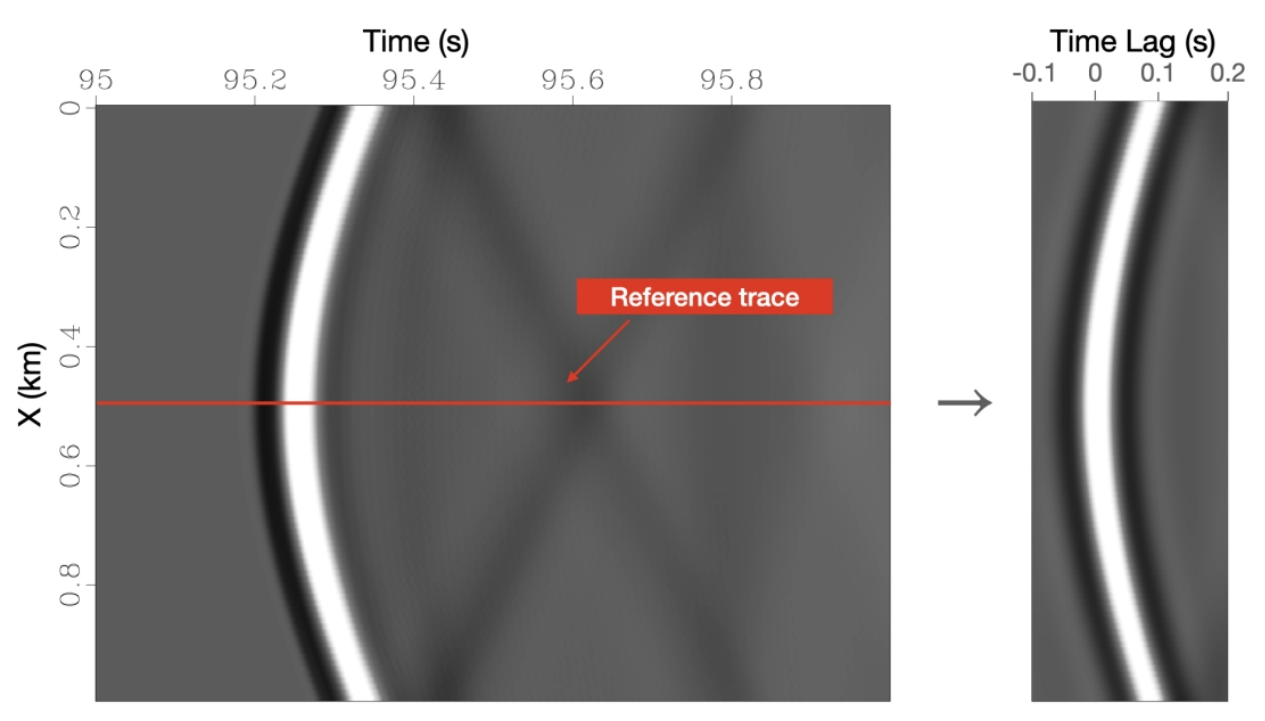

Fig. 1, Data before and after the correlation operation.

$677 \times 381 \mathrm{~mm}(72 \times 72 \mathrm{DPI})$ 


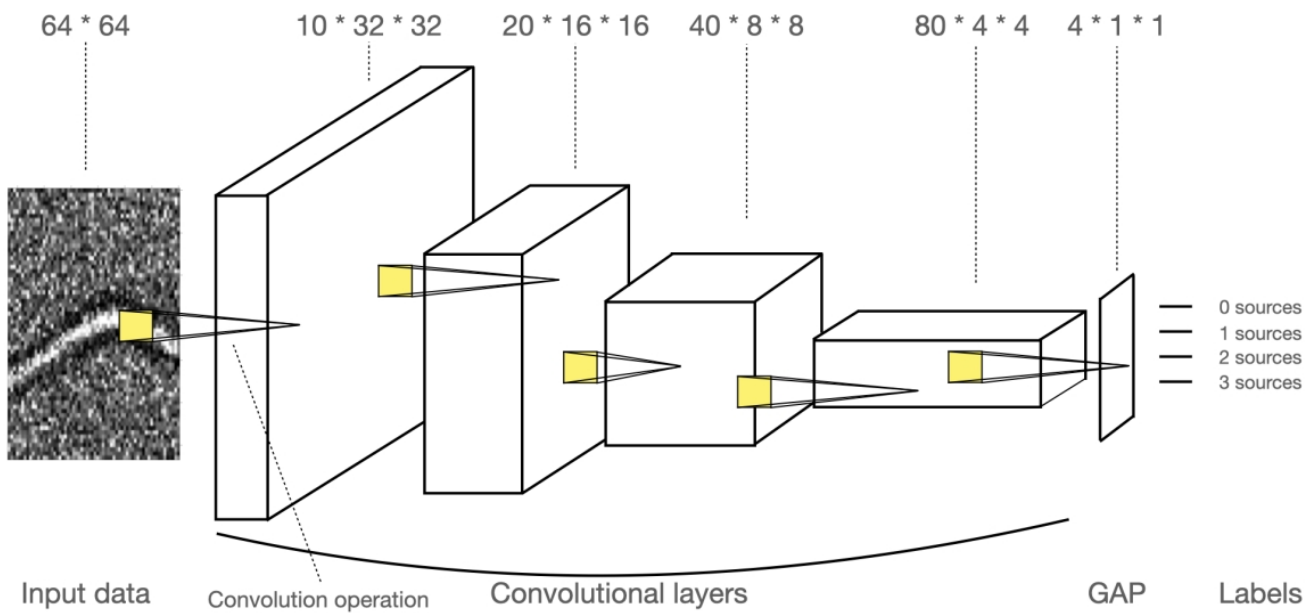

Fig. 2, CNN with a global average pooling layer for solving classification issues. The yellow blocks refer to convolutional operators. The numbers on the top refer to the operator sizes and the data sizes. The numbers of each convolutional layer size indicate the output channels * output data size in the first dimension* output data size in the second dimension.

$677 \times 381 \mathrm{~mm}(72 \times 72 \mathrm{DPI})$ 


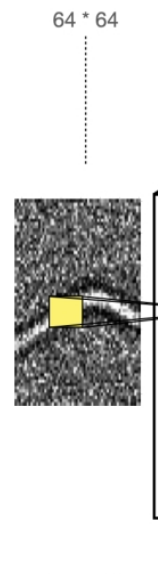

$3 * 32 * 32 \quad 6 * 16 * 16 \quad 12 * 8 * 8 \quad 24 * 4 * 4$

$48 * 2 * 2$

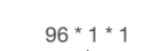

$96 \quad 2012$

Input data

Convolution operation Convolutional layers

Fully connected laters

Labels

Fig. 3, CNN with fully connected layers for solving regression issues. The yellow blocks refer to convolutional operators. The numbers on the top refer to the operator sizes and the data sizes that indicate the output channels * output data size in the first dimension * output data size in the second dimension.

\section{$677 \times 381 \mathrm{~mm}(72 \times 72 \mathrm{DPI})$}




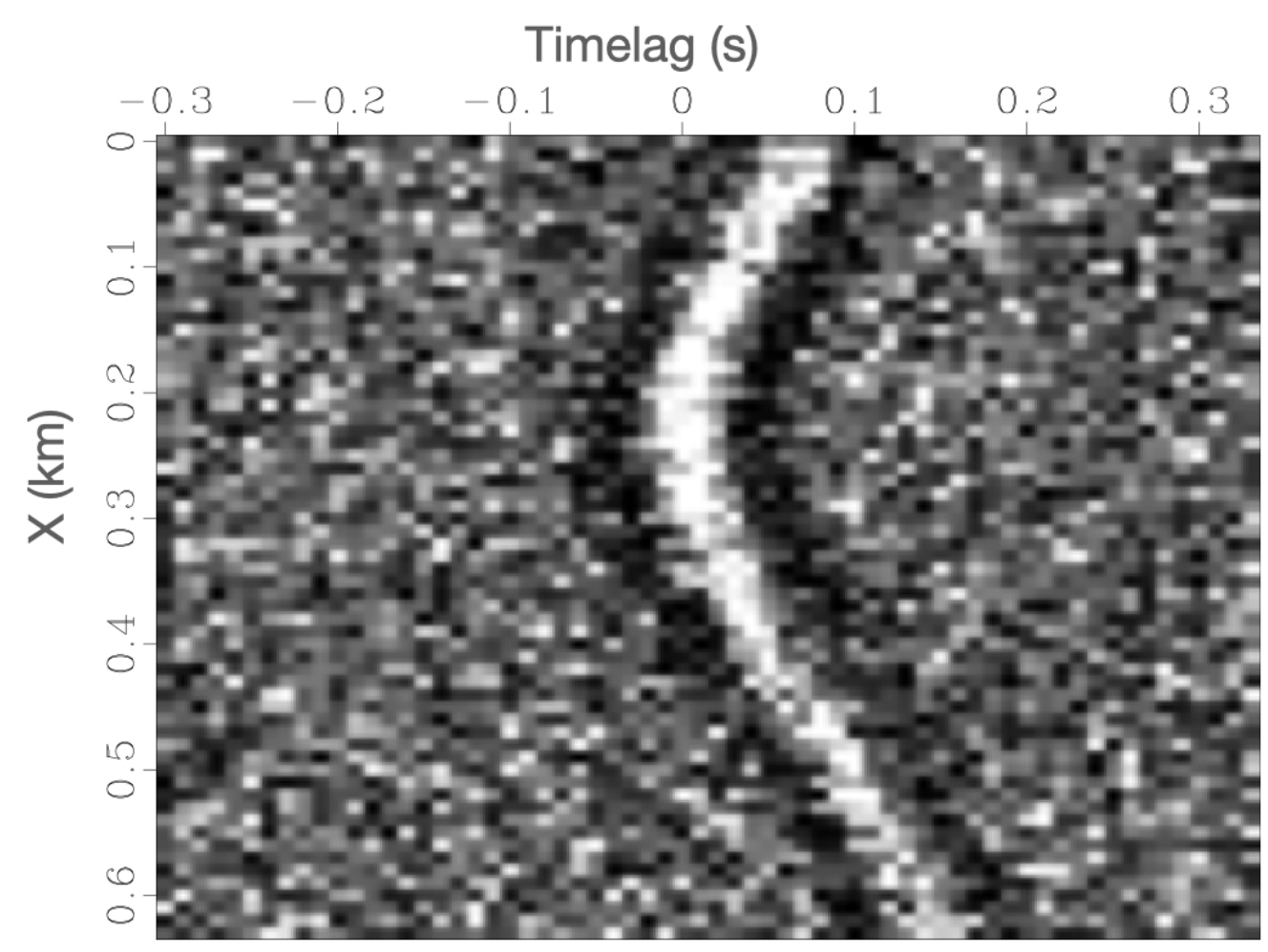

Fig. 4a, Example correlated data with (a) single (b) double (c) triple (d) zero events. Random noise of $\$ 50 \backslash \% \$$ level has been added.

$361 \times 270 \mathrm{~mm}(72 \times 72 \mathrm{DPI})$ 


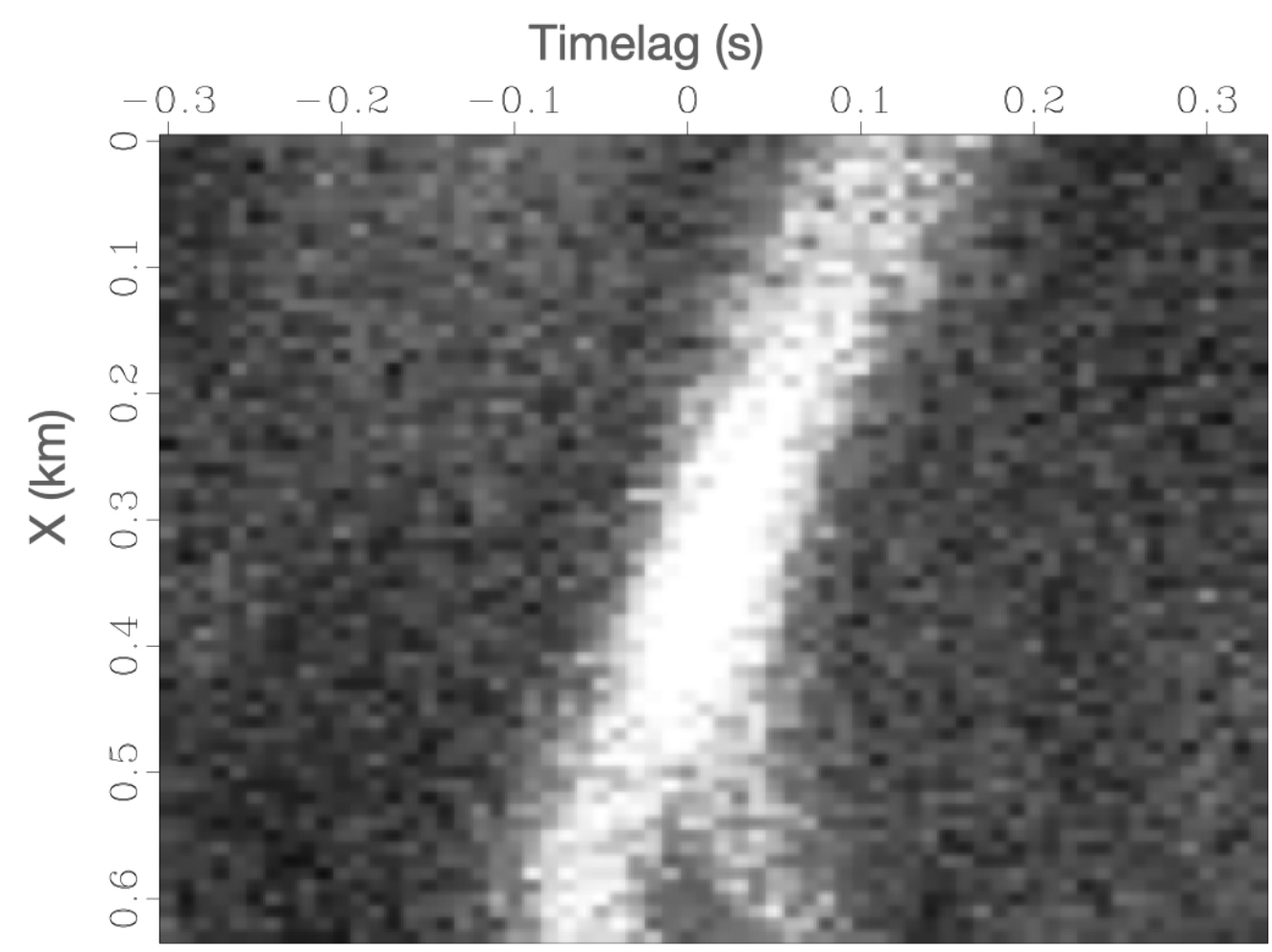

Fig. 4b, Example correlated data with (a) single (b) double (c) triple (d) zero events. Random noise of $\$ 50 \backslash \% \$$ level has been added.

$361 \times 270 \mathrm{~mm}(72 \times 72 \mathrm{DPI})$ 


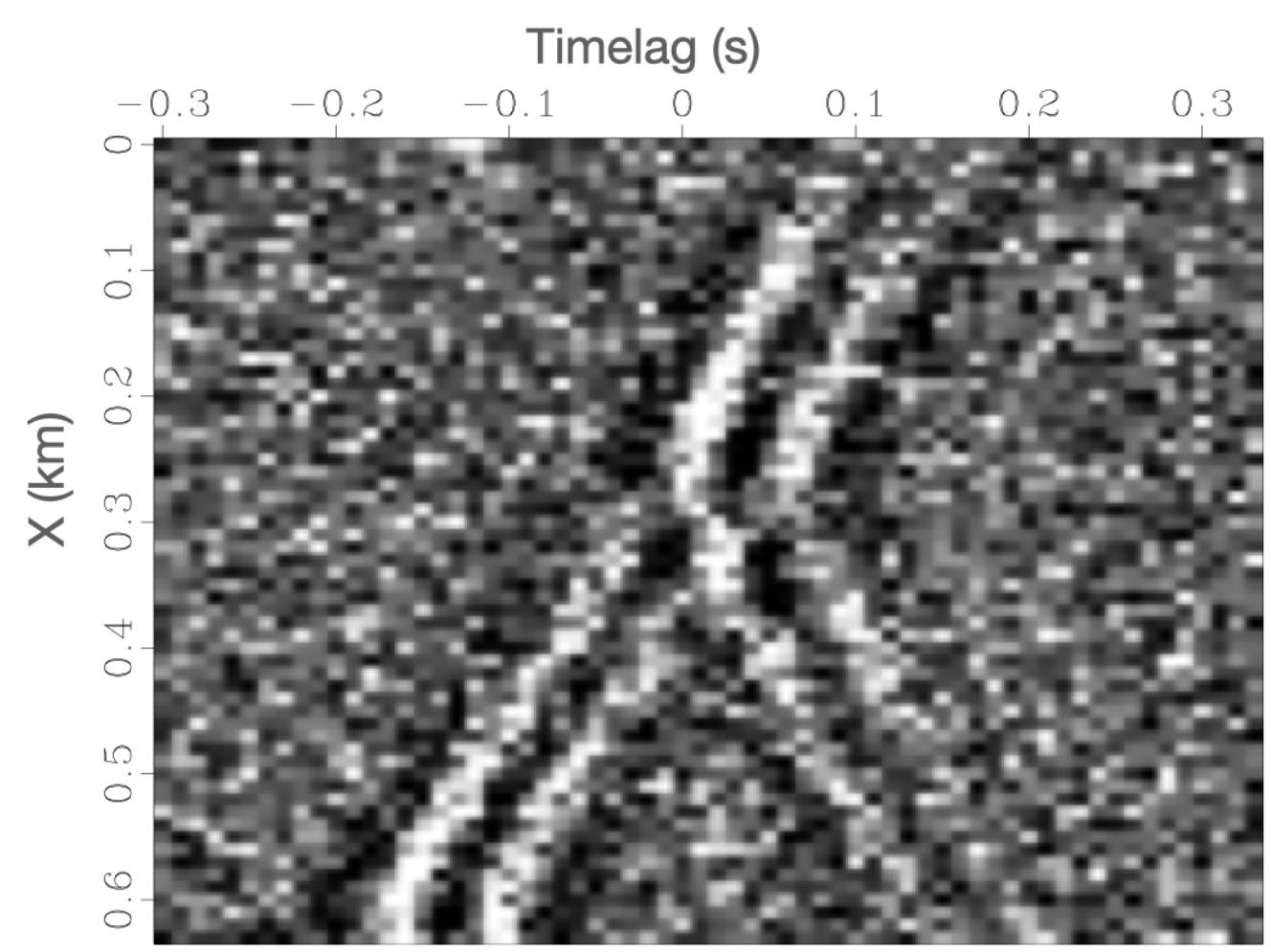

Fig. 4c, Example correlated data with (a) single (b) double (c) triple (d) zero events. Random noise of $\$ 50 \backslash \% \$$ level has been added.

$361 \times 270 \mathrm{~mm}(72 \times 72 \mathrm{DPI})$

This paper presented here as accepted for publication in Geophysics prior to copyediting and composition.

(C) 2021 Society of Exploration Geophysicists 


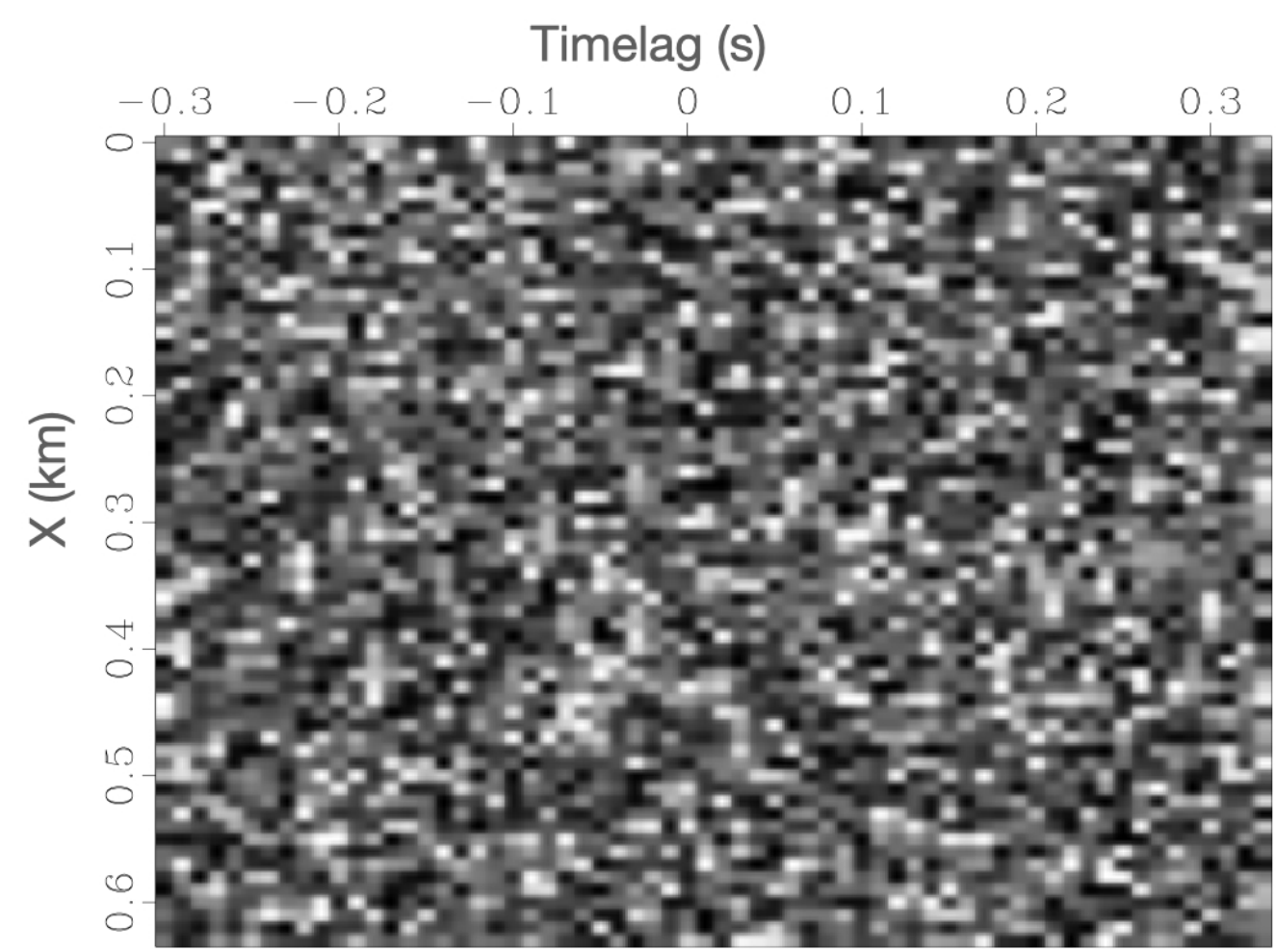

Fig. 4d, Example correlated data with (a) single (b) double (c) triple (d) zero events. Random noise of $\$ 50 \backslash \% \$$ level has been added.

$361 \times 270 \mathrm{~mm}(72 \times 72 \mathrm{DPI})$ 


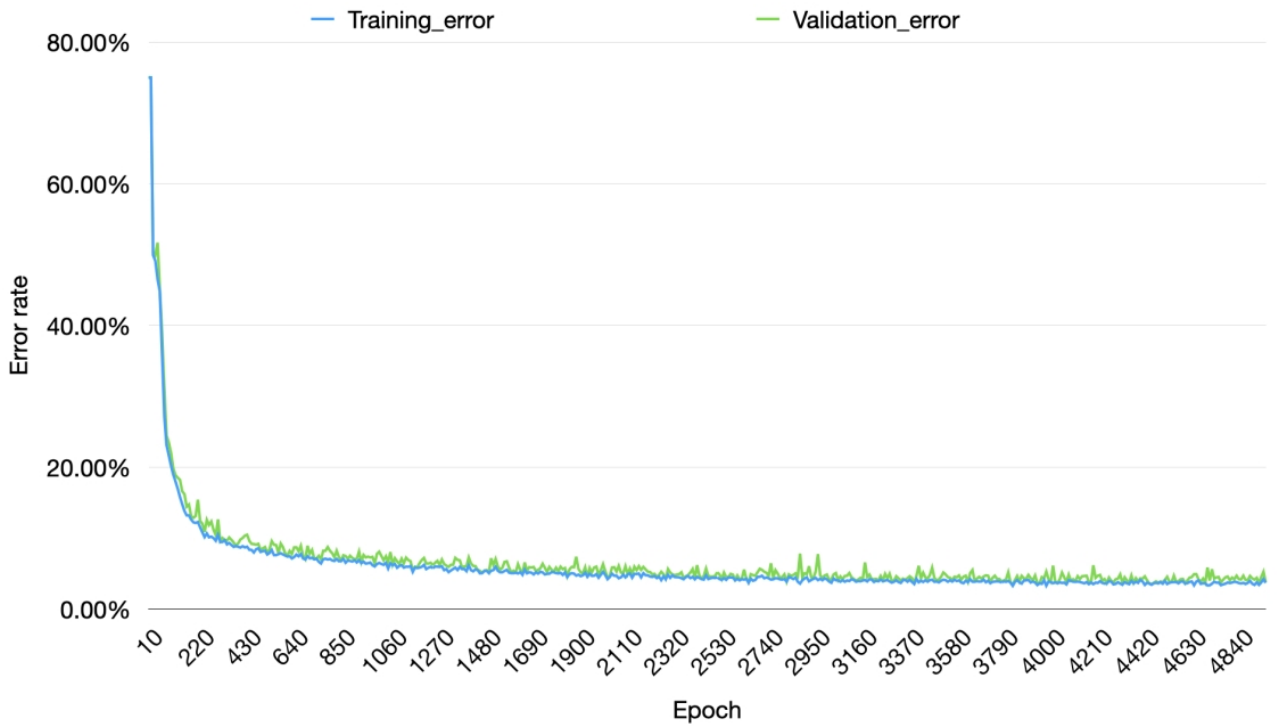

Fig. 5a, (a) Training (blue) and validation/testing (green) error rate curves for classification; (b) Training (blue) and validation/testing (green) loss curves for regression.

$677 \times 381 \mathrm{~mm}(72 \times 72 \mathrm{DPI})$ 

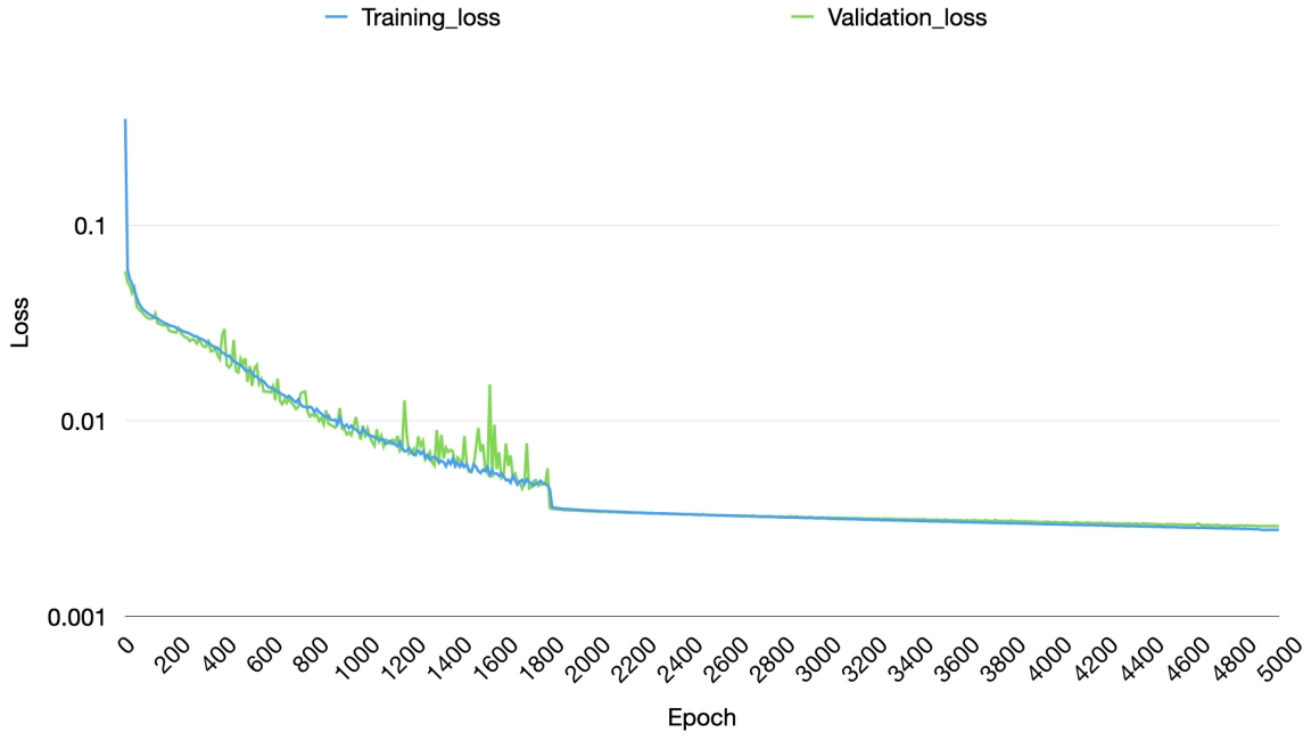

Fig. 5b, (a) Training (blue) and validation/testing (green) error rate curves for classification; (b) Training (blue) and validation/testing (green) loss curves for regression.

$677 \times 381 \mathrm{~mm}(72 \times 72$ DPI $)$ 


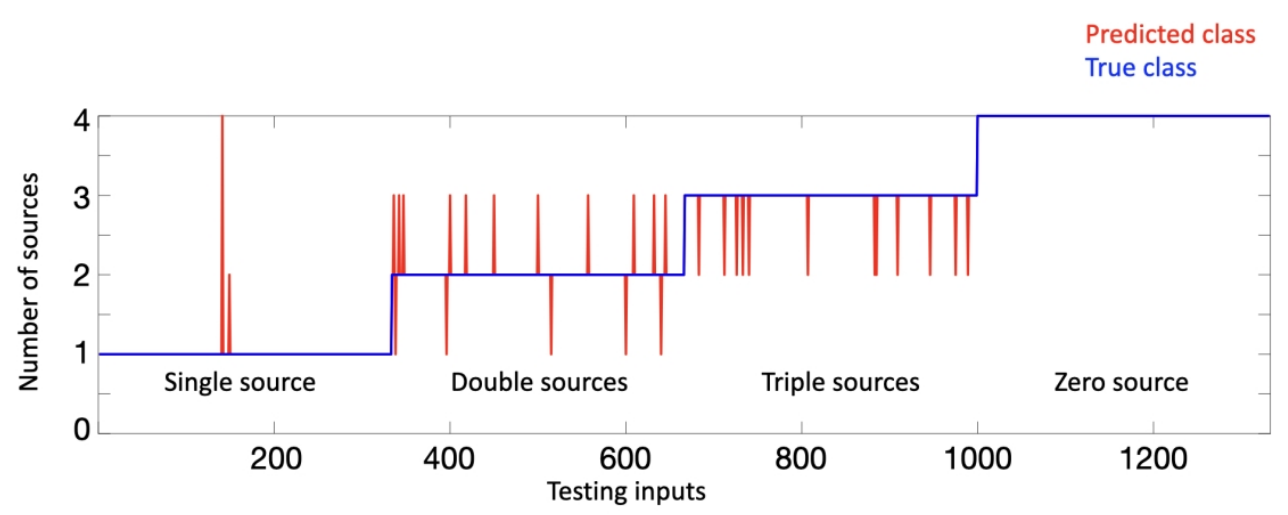

Fig. 6, The predicted (red) and the true source numbers $\$ N \$$ in each testing data.

$677 \times 282 \mathrm{~mm}(72 \times 72 \mathrm{DPI})$

This paper presented here as accepted for publication in Geophysics prior to copyediting and composition.

(C) 2021 Society of Exploration Geophysicists 


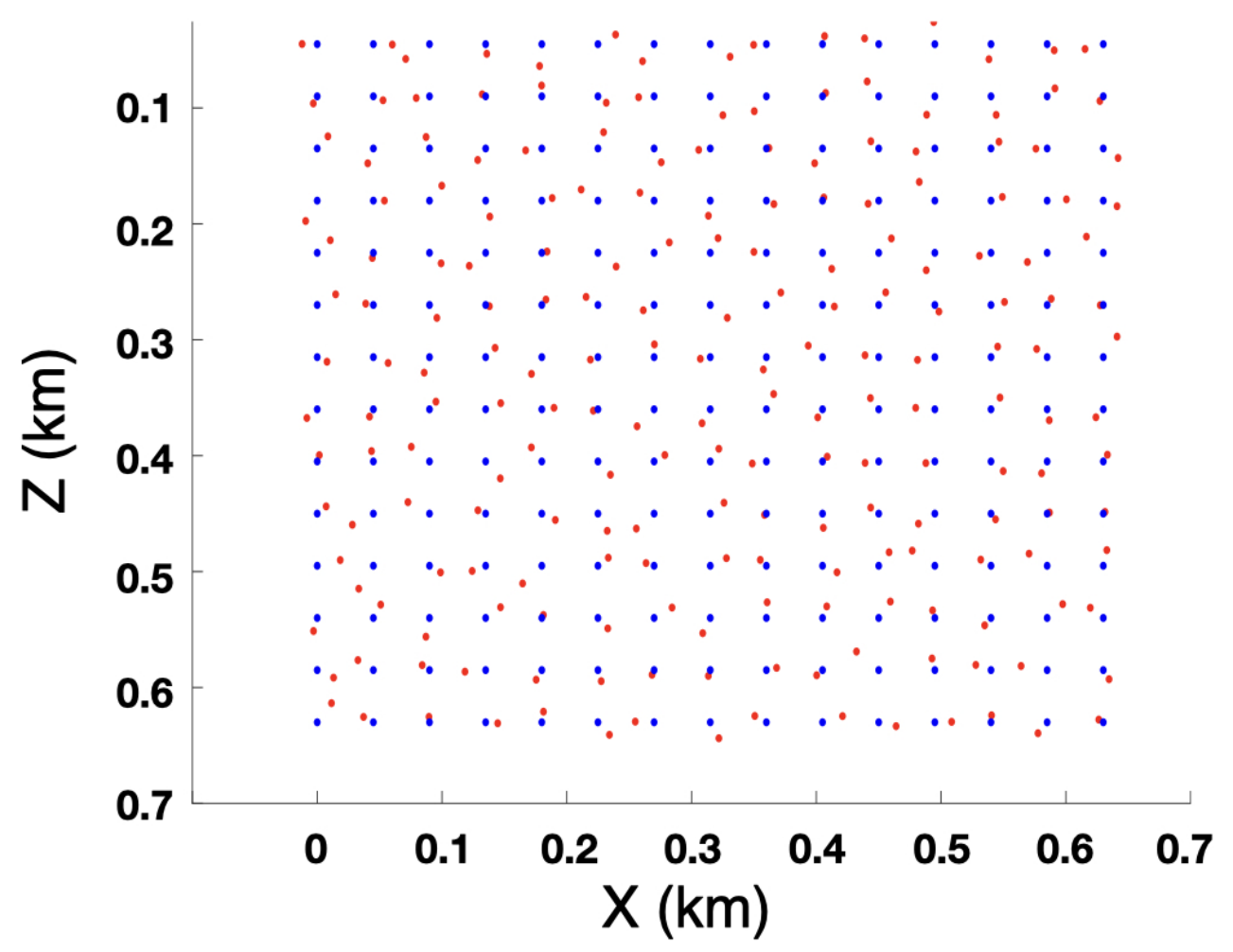

Fig. 7a, $\$ V(z) \$$ model (a) single (c) double, and (e) triple sources cases coordinates $\$ X \backslash \& Z \$$ predictions. Corresponding error maps for (b) single (d) double, and (f) triple sources cases. The yellow color indicates large errors in space, and the blue color indicates small errors. The red dots indicate all the event locations in the multi-events data.

$361 \times 270 \mathrm{~mm}(72 \times 72 \mathrm{DPI})$ 


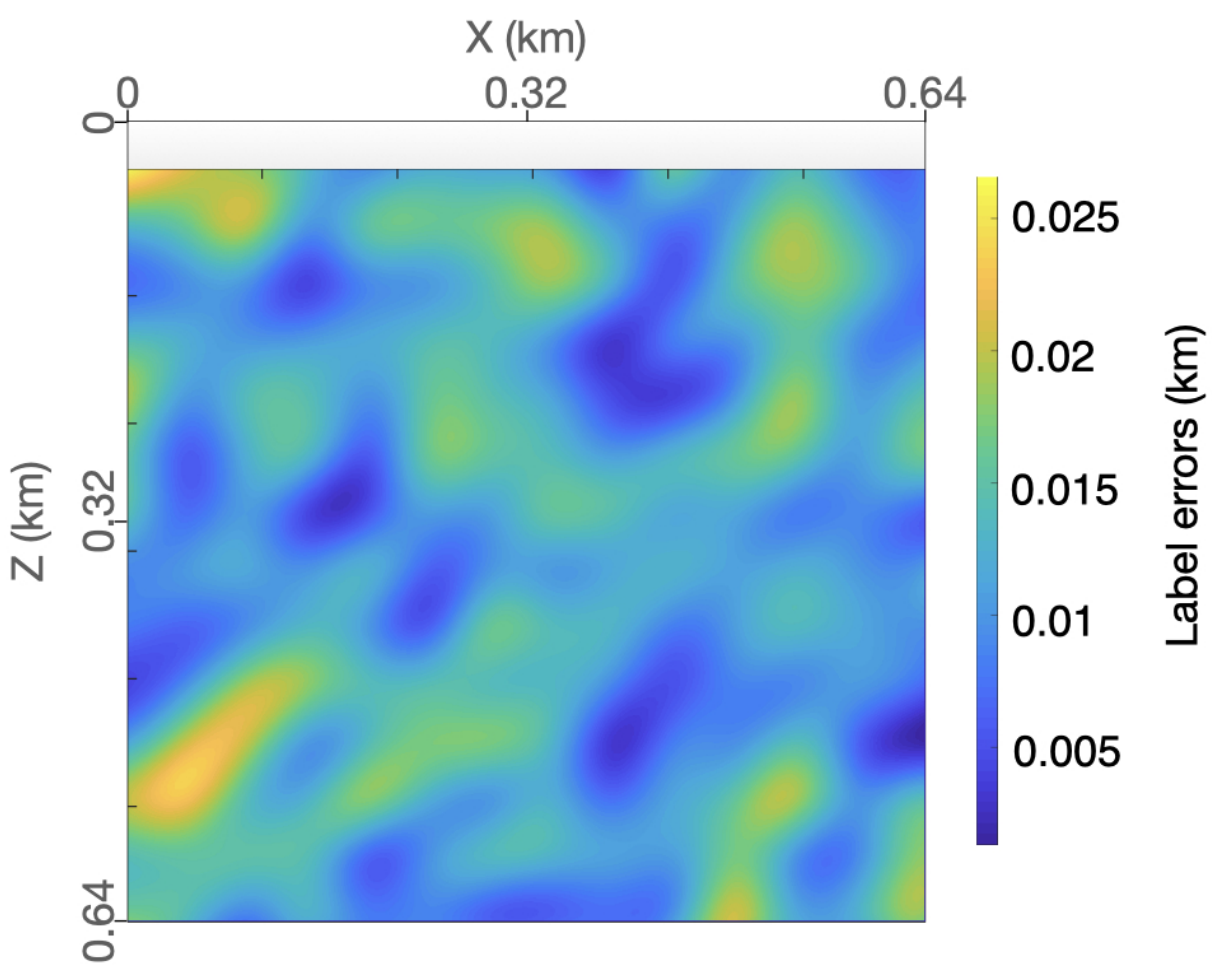

Fig. $7 b, \$ V(z) \$$ model (a) single (c) double, and (e) triple sources cases coordinates $\$ X \backslash \& Z \$$ predictions. Corresponding error maps for (b) single (d) double, and (f) triple sources cases. The yellow color indicates large errors in space, and the blue color indicates small errors. The red dots indicate all the event locations in the multi-events data.

$361 \times 270 \mathrm{~mm}(72 \times 72 \mathrm{DPI})$ 


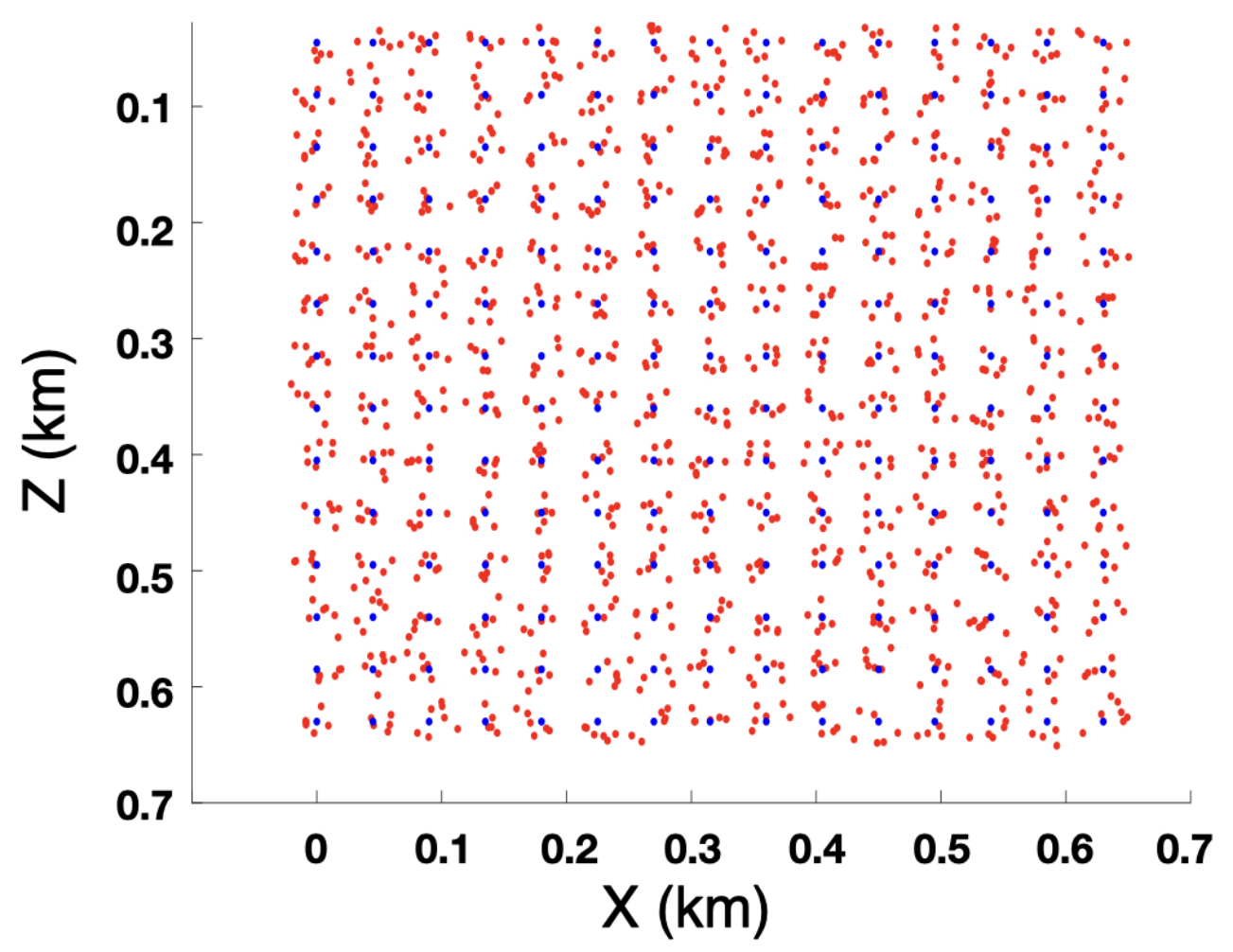

Fig. 7c, $\$ V(z) \$$ model (a) single (c) double, and (e) triple sources cases coordinates $\$ X \backslash \& Z \$$ predictions. Corresponding error maps for (b) single (d) double, and (f) triple sources cases. The yellow color indicates large errors in space, and the blue color indicates small errors. The red dots indicate all the event locations in the multi-events data.

$361 \times 270 \mathrm{~mm}(72 \times 72 \mathrm{DPI})$ 


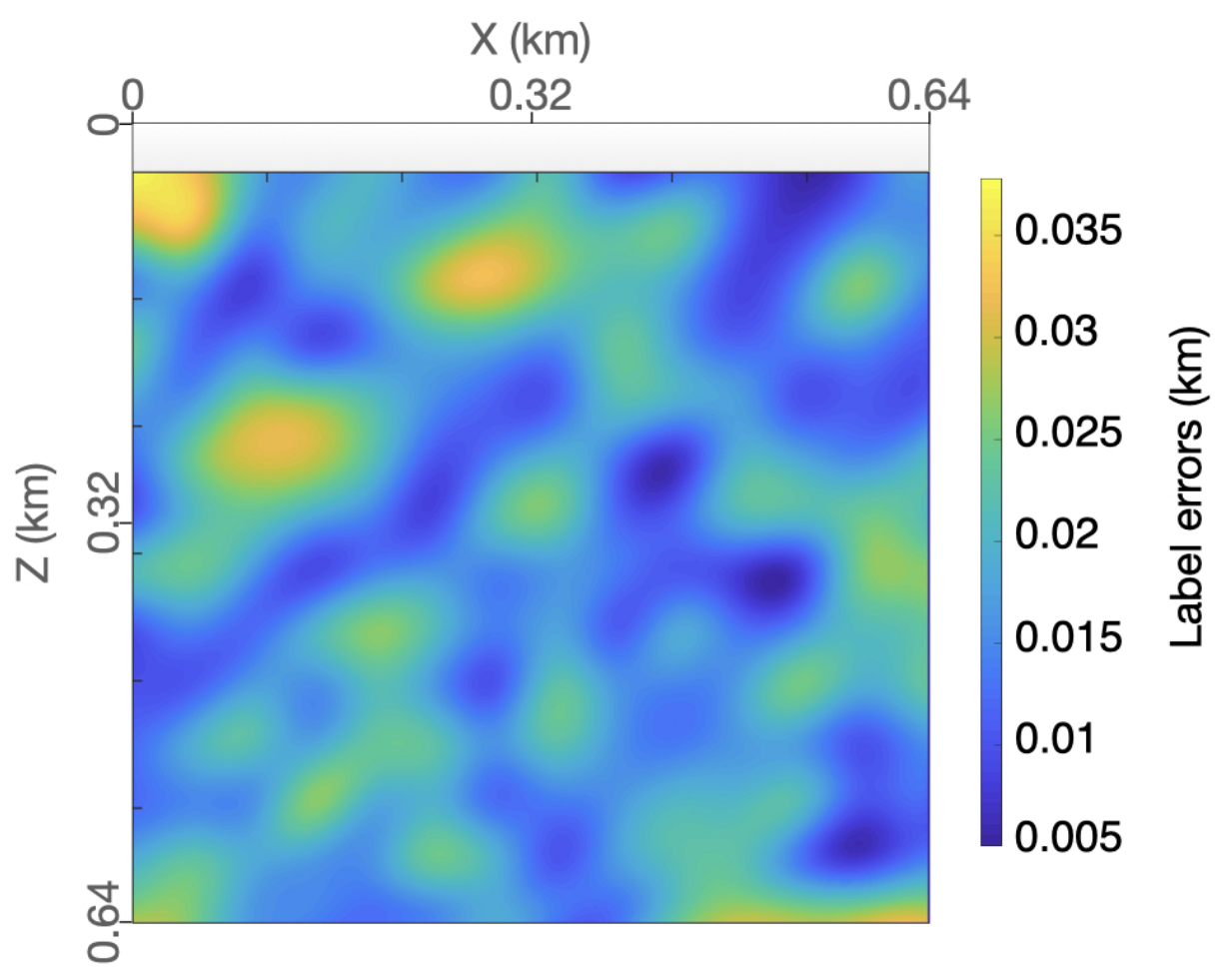

Fig. 7d, $\$ V(z) \$$ model (a) single (c) double, and (e) triple sources cases coordinates $\$ X \backslash \& Z \$$ predictions. Corresponding error maps for (b) single (d) double, and (f) triple sources cases. The yellow color indicates large errors in space, and the blue color indicates small errors. The red dots indicate all the event locations in the multi-events data.

$361 \times 270 \mathrm{~mm}(72 \times 72 \mathrm{DPI})$ 


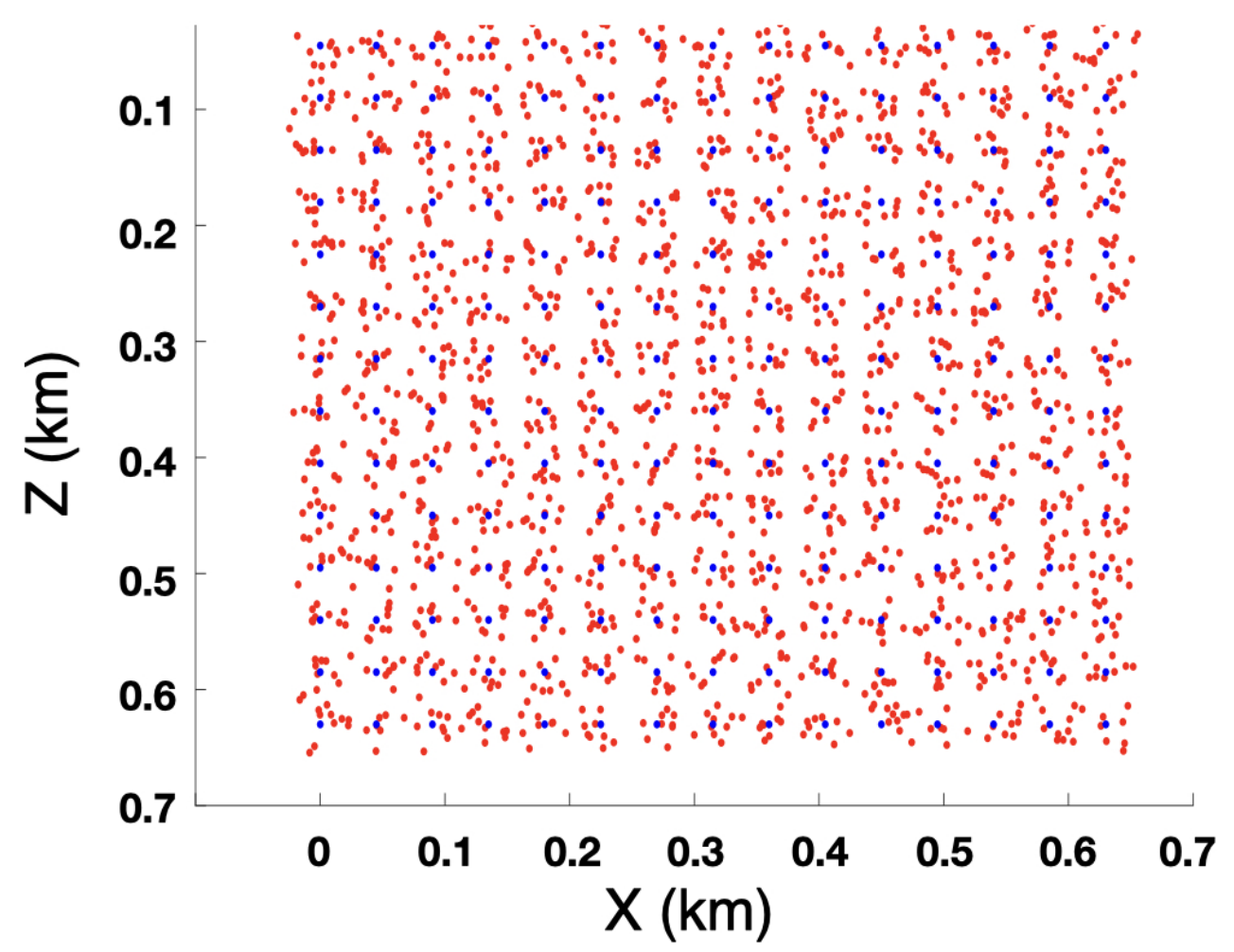

Fig. 7e, $\$ V(z) \$$ model (a) single (c) double, and (e) triple sources cases coordinates $\$ X \backslash \& Z \$$ predictions. Corresponding error maps for (b) single (d) double, and (f) triple sources cases. The yellow color indicates large errors in space, and the blue color indicates small errors. The red dots indicate all the event locations in the multi-events data.

$361 \times 270 \mathrm{~mm}(72 \times 72 \mathrm{DPI})$ 


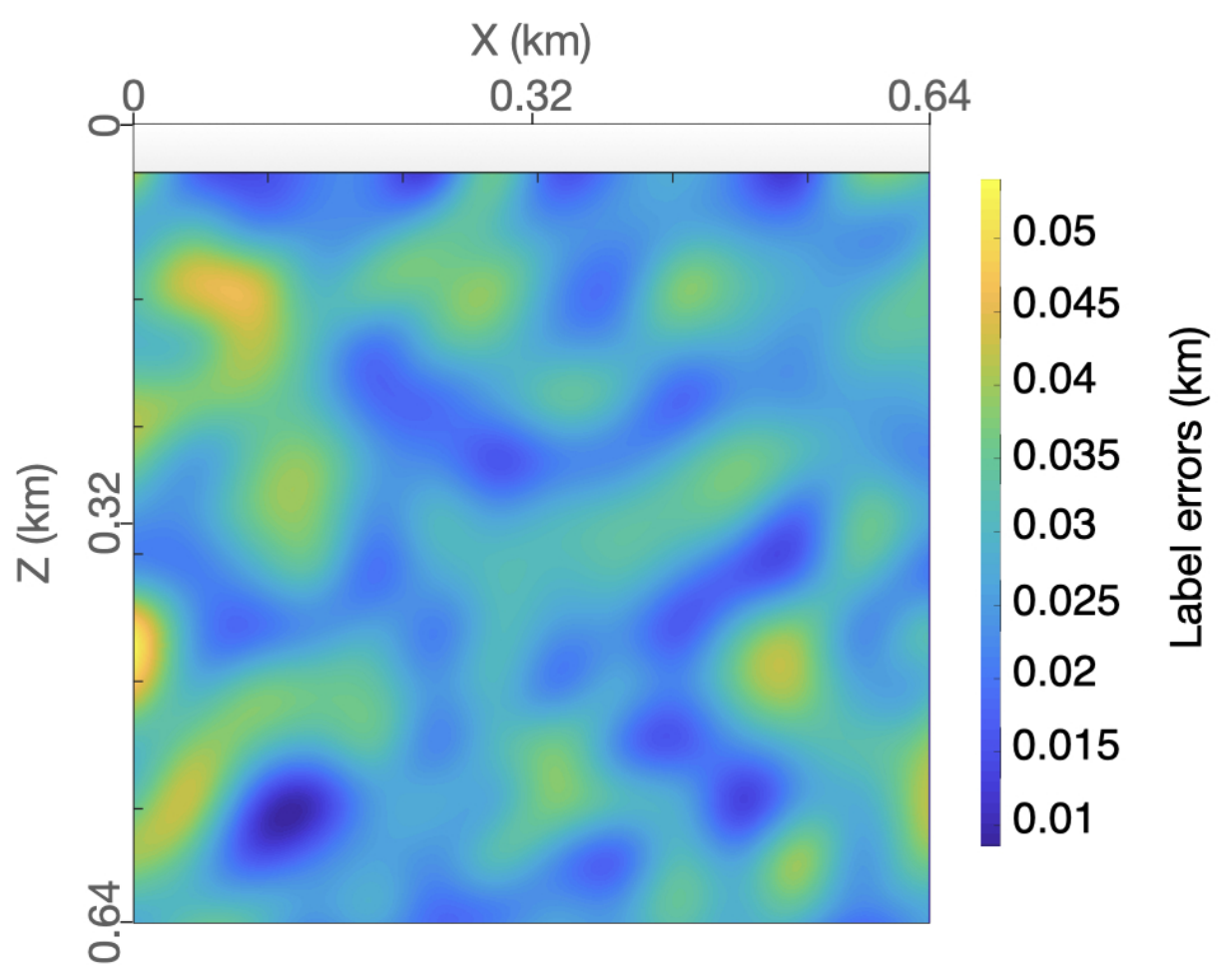

Fig. 7f, $\$ V(z) \$$ model (a) single (c) double, and (e) triple sources cases coordinates $\$ X \backslash \& Z \$$ predictions. Corresponding error maps for (b) single (d) double, and (f) triple sources cases. The yellow color indicates large errors in space, and the blue color indicates small errors. The red dots indicate all the event locations in the multi-events data.

$361 \times 270 \mathrm{~mm}(72 \times 72 \mathrm{DPI})$ 

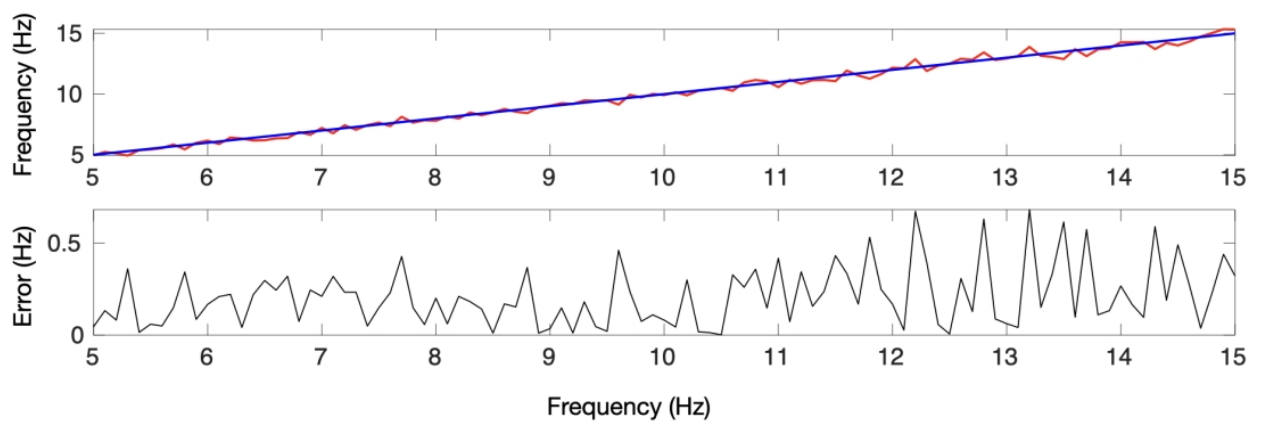

Fig. 8a, Predicted source frequencies $\$ F \$$ for (a) single (b) double (c) triple sources cases. $\$ 100 \$$ frequencies are shown for each case. True and predicted labels are shown as the blue and red curves, respectively.

$677 \times 246 \mathrm{~mm}(72 \times 72 \mathrm{DPI})$ 

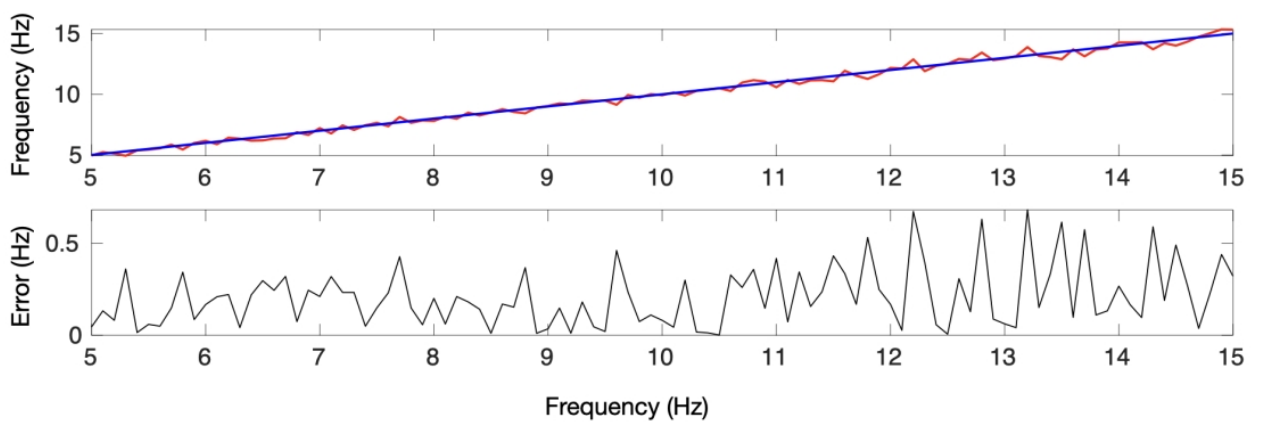

Fig. 8b, Predicted source frequencies $\$ F \$$ for (a) single (b) double (c) triple sources cases. $\$ 100 \$$ frequencies are shown for each case. True and predicted labels are shown as the blue and red curves, respectively.

$677 \times 246 \mathrm{~mm}(72 \times 72 \mathrm{DPI})$

This paper presented here as accepted for publication in Geophysics prior to copyediting and composition.

(C) 2021 Society of Exploration Geophysicists 

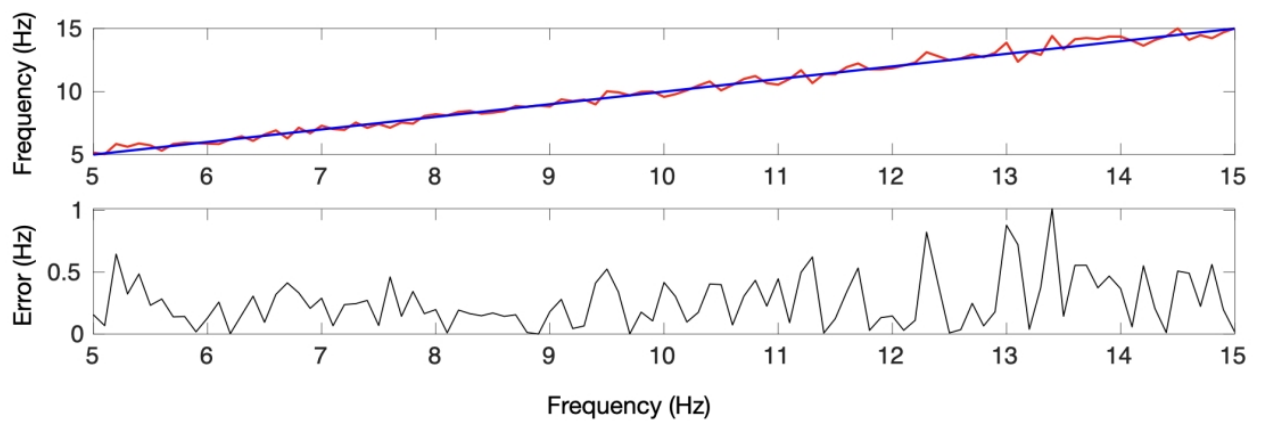

Fig. 8c, Predicted source frequencies $\$ F \$$ for (a) single (b) double (c) triple sources cases. $\$ 100 \$$ frequencies are shown for each case. True and predicted labels are shown as the blue and red curves, respectively.

\section{$677 \times 246 \mathrm{~mm}(72 \times 72 \mathrm{DPI})$}



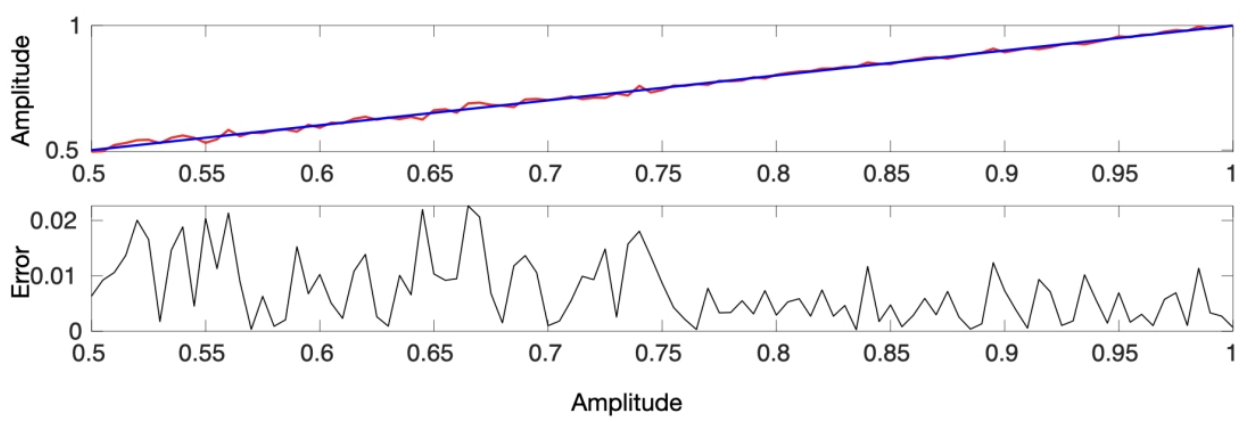

Fig. 9a, Predicted source amplitudes $\$ A \$$ for (a) single (b) double (c) triple sources cases. $\$ 100 \$$ examples are shown for each case. True and predicted labels are shown as the blue and red curves, respectively.

$677 \times 246 \mathrm{~mm}(72 \times 72 \mathrm{DPI})$ 

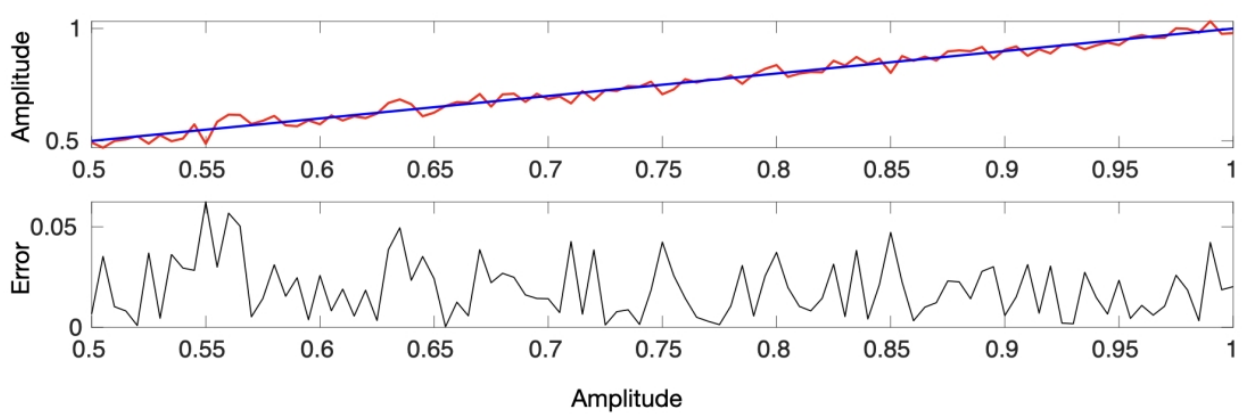

Fig. 9b, Predicted source amplitudes $\$ A \$$ for (a) single (b) double (c) triple sources cases. $\$ 100 \$$ examples are shown for each case. True and predicted labels are shown as the blue and red curves, respectively.

$677 \times 246 \mathrm{~mm}(72 \times 72 \mathrm{DPI})$ 

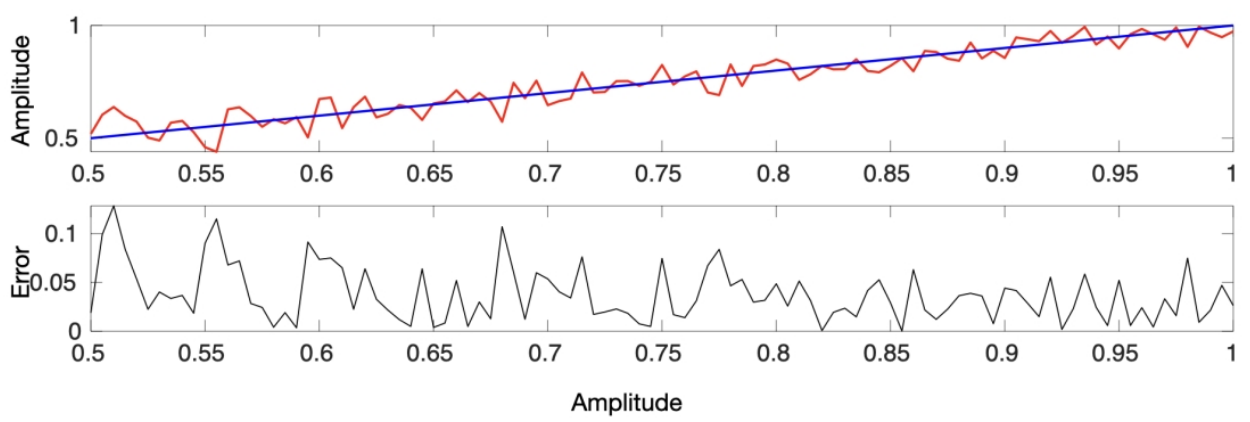

Fig. 9c, Predicted source amplitudes $\$ A \$$ for (a) single (b) double (c) triple sources cases. $\$ 100 \$$ examples are shown for each case. True and predicted labels are shown as the blue and red curves, respectively.

$677 \times 246 \mathrm{~mm}(72 \times 72 \mathrm{DPI})$

This paper presented here as accepted for publication in Geophysics prior to copyediting and composition.

(C) 2021 Society of Exploration Geophysicists 


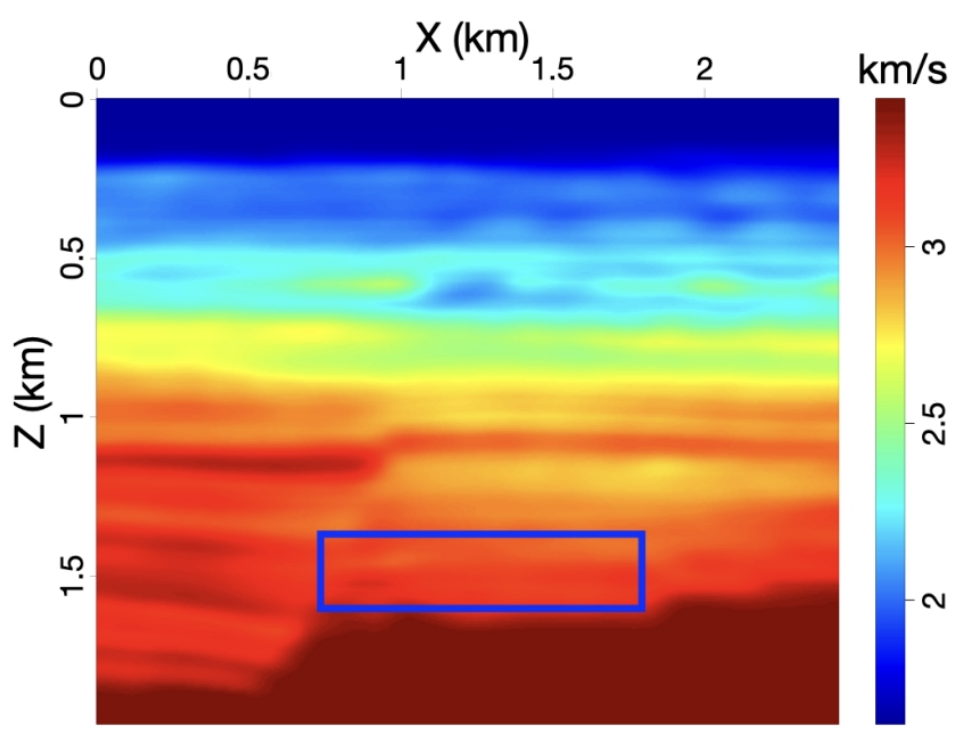

Fig. 10a, (a) Smoothed (MVA) Otway P-wave velocity model used for generating the training set. (b) True model used for generating the testing set on regular grids.

$493 \times 270 \mathrm{~mm}(72 \times 72 \mathrm{DPI})$ 


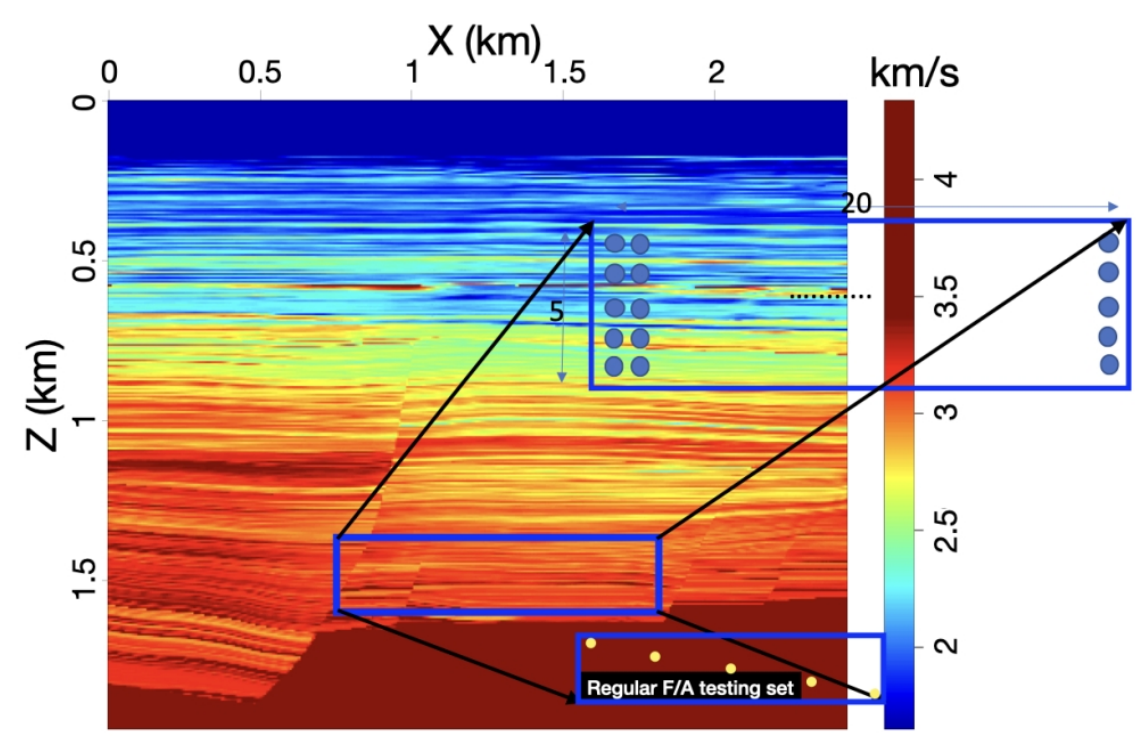

Fig. 10b, (a) Smoothed (MVA) Otway P-wave velocity model used for generating the training set. (b) True model used for generating the testing set on regular grids.

$493 \times 270 \mathrm{~mm}(72 \times 72$ DPI $)$ 

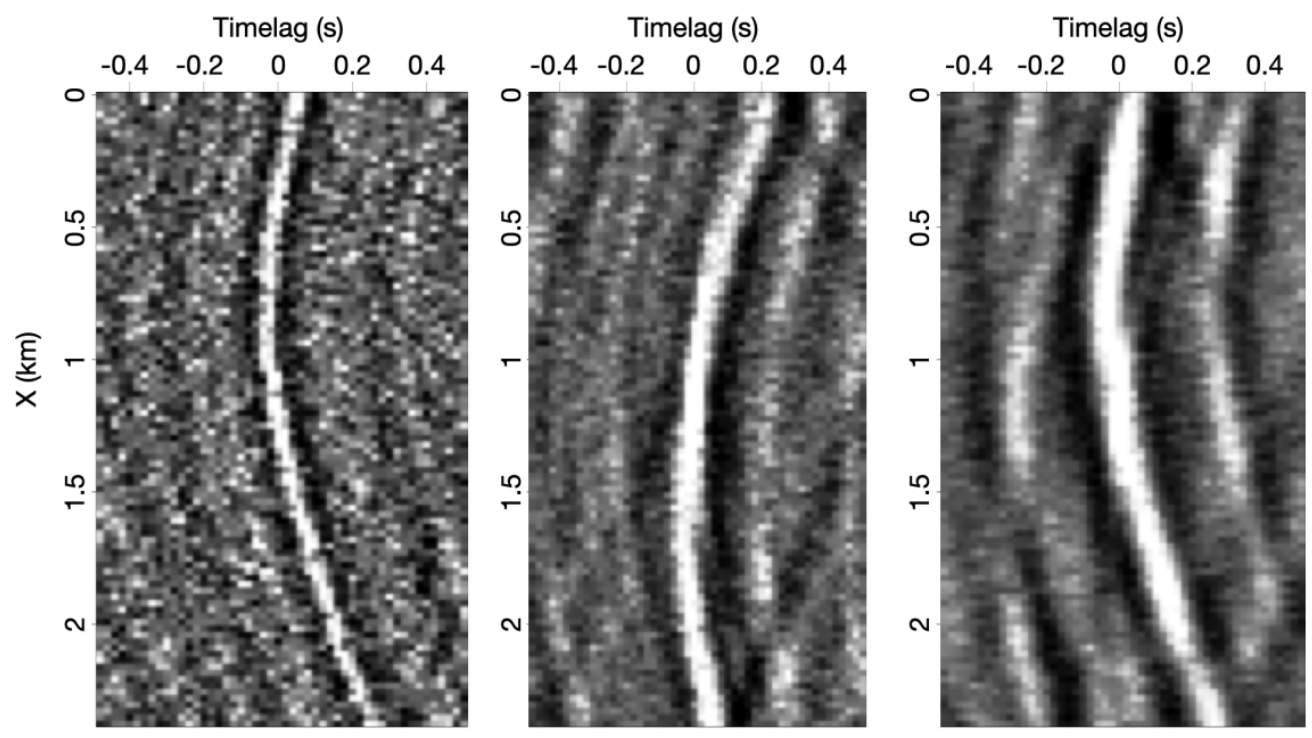

Fig. 11a, Non-zero example correlated data in (a) training (b) testing set with (left) one (middle) two (right) three sources. Random noise of $\$ 50 \backslash \% \$$ level has been added.

$677 \times 381 \mathrm{~mm}(72 \times 72$ DPI $)$ 

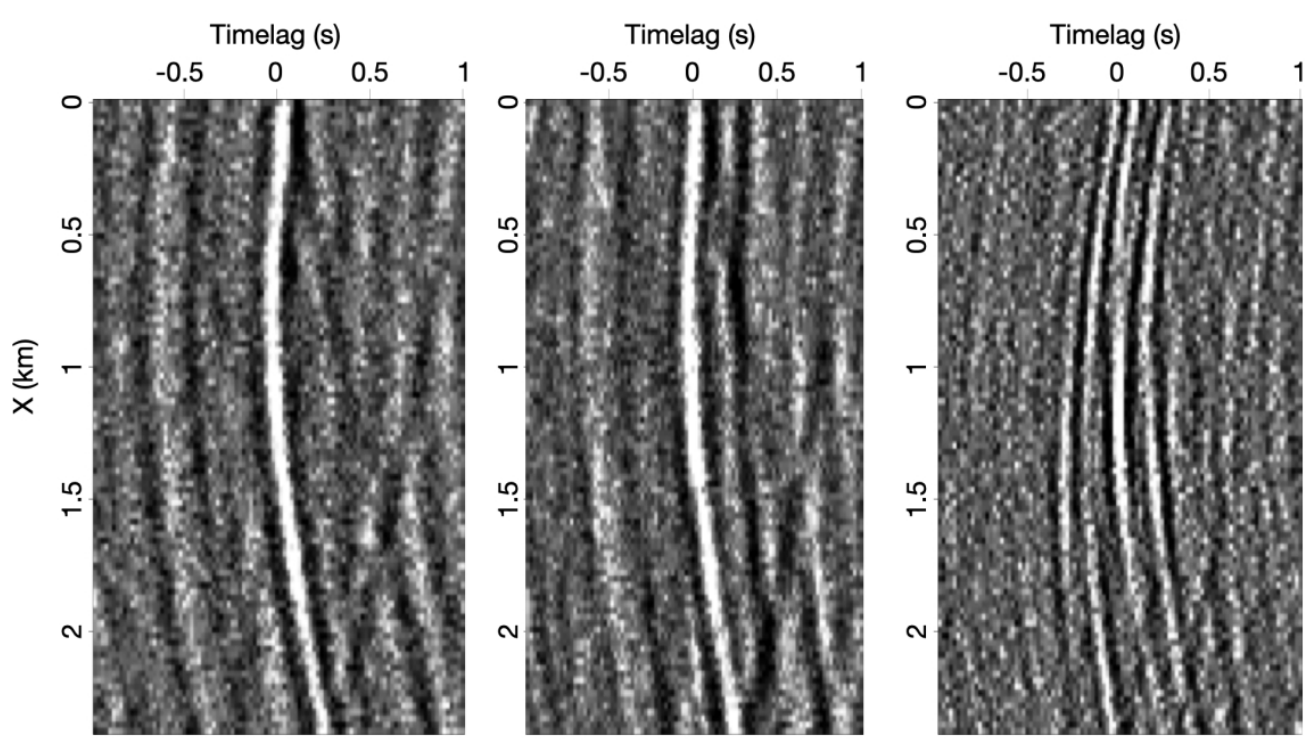

Fig. 11b, Non-zero example correlated data in (a) training (b) testing set with (left) one (middle) two (right) three sources. Random noise of $\$ 50 \backslash \% \$$ level has been added.

$677 \times 381 \mathrm{~mm}(72 \times 72 \mathrm{DPI})$ 


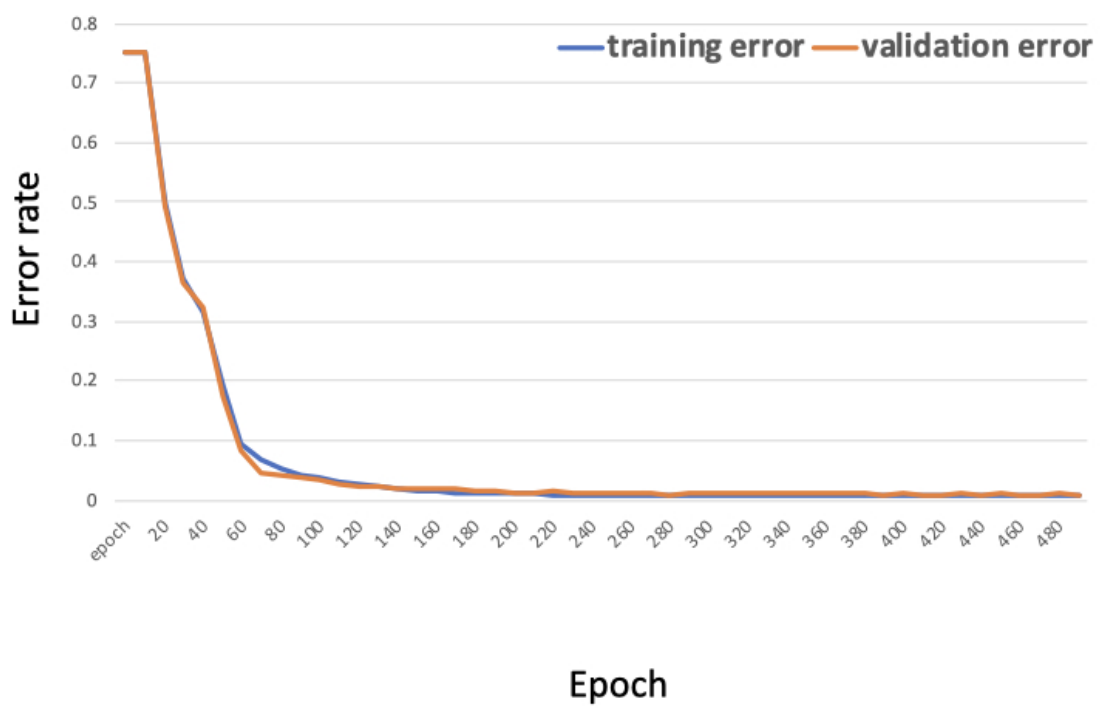

Fig. 12a, (a) Classification and (b) regression training and validation loss curves.

$297 \times 209 \mathrm{~mm}(72 \times 72 \mathrm{DPI})$

This paper presented here as accepted for publication in Geophysics prior to copyediting and composition.

(C) 2021 Society of Exploration Geophysicists 


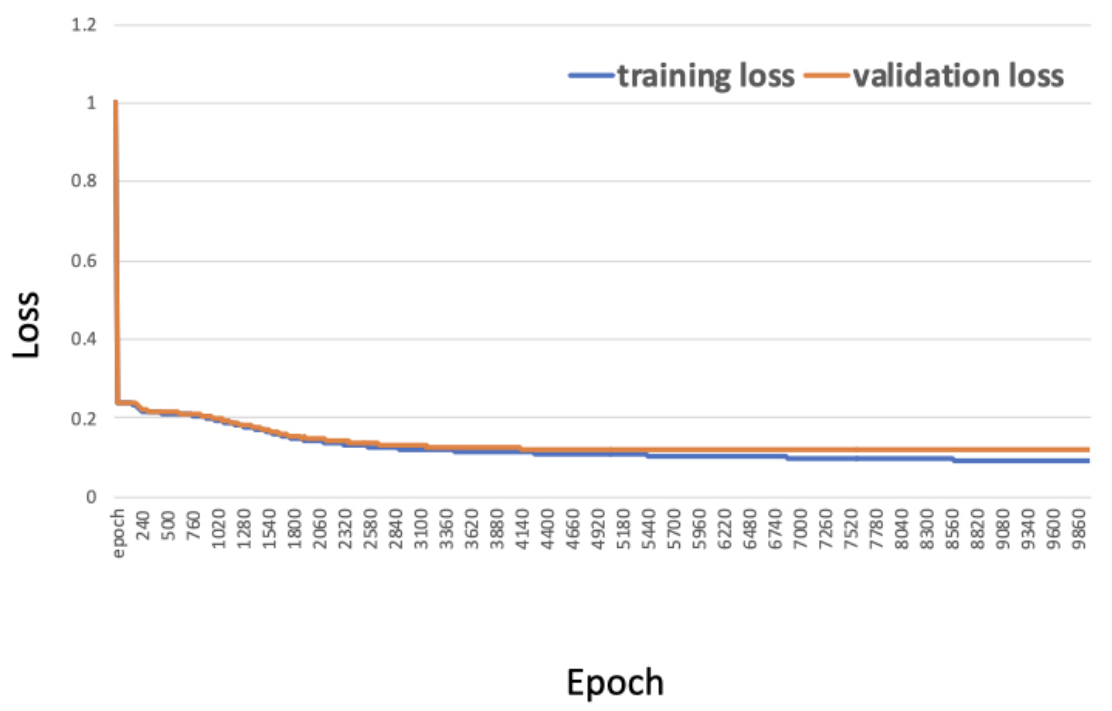

Fig. 12b, (a) Classification and (b) regression training and validation loss curves.

$297 \times 209 \mathrm{~mm}(72 \times 72 \mathrm{DPI})$

This paper presented here as accepted for publication in Geophysics prior to copyediting and composition.

(C) 2021 Society of Exploration Geophysicists 


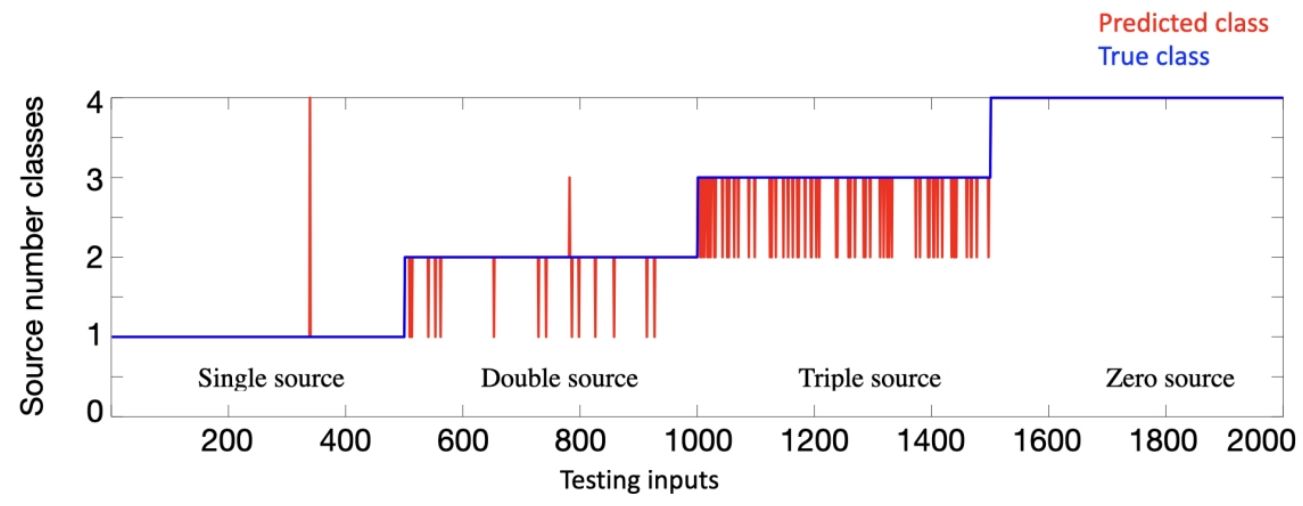

Fig. 13, The predicted (red) and the true source numbers $\$ N \$$ in each testing data on the Otway model.

$677 \times 282 \mathrm{~mm}(72 \times 72 \mathrm{DPI})$ 


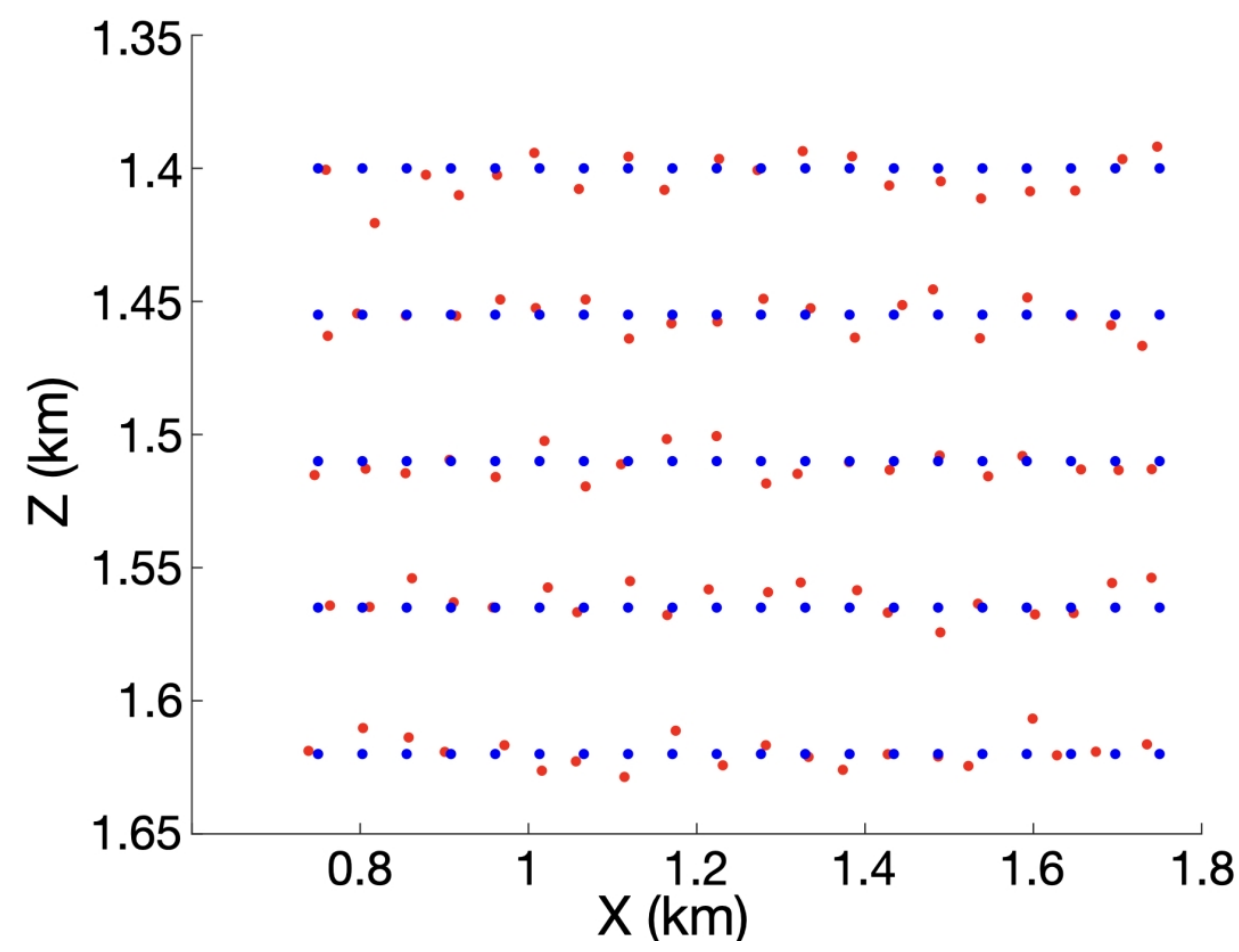

Fig. 14a, Otway model (a) single (c) double, and (e) triple sources cases coordinates $\$ X \backslash \& Z \$$ predictions. Corresponding error maps for (b) single (d) double, and (f) triple sources cases. The yellow color indicates larger errors in space and the blue color indicates smaller errors. The red dots indicate all the event locations in the multi events data.

$677 \times 508 \mathrm{~mm}(72 \times 72 \mathrm{DPI})$ 


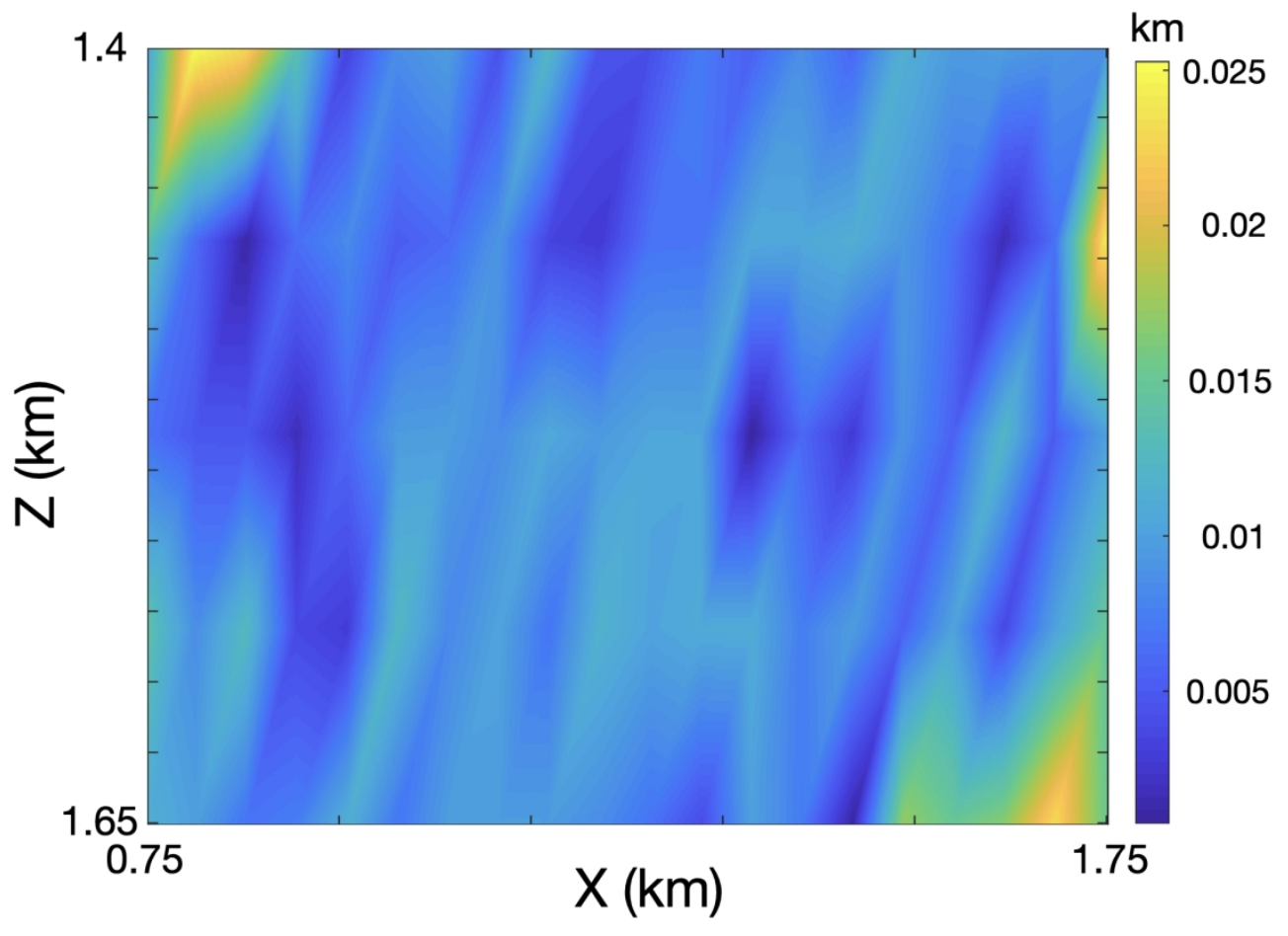

Fig. 14b, Otway model (a) single (c) double, and (e) triple sources cases coordinates $\$ X \backslash \& Z \$$ predictions. Corresponding error maps for (b) single (d) double, and (f) triple sources cases. The yellow color indicates larger errors in space and the blue color indicates smaller errors. The red dots indicate all the event locations in the multi events data.

$677 \times 508 \mathrm{~mm}(72 \times 72 \mathrm{DPI})$ 


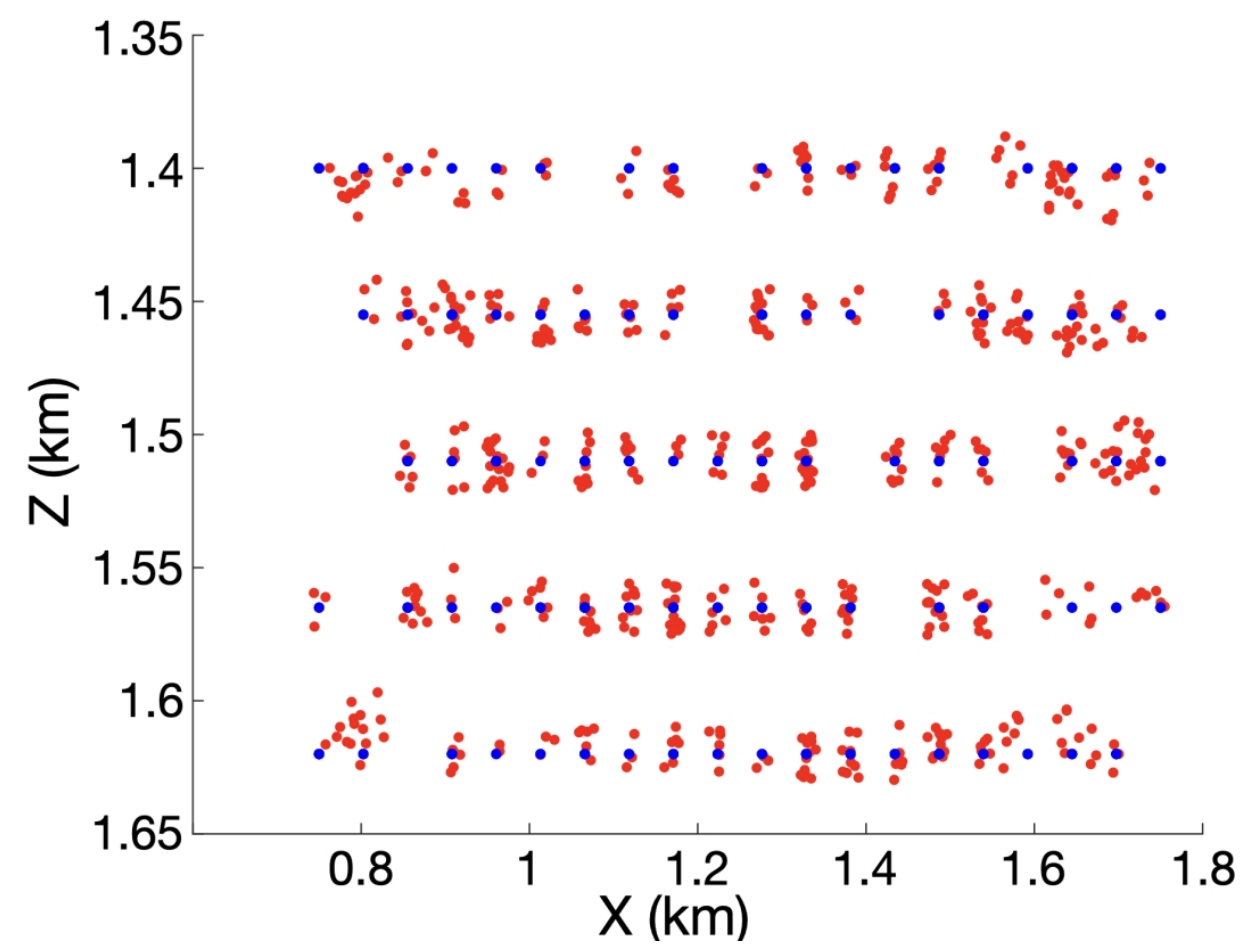

Fig. 14c, Otway model (a) single (c) double, and (e) triple sources cases coordinates $\$ X \backslash \& Z \$$ predictions. Corresponding error maps for (b) single (d) double, and (f) triple sources cases. The yellow color indicates larger errors in space and the blue color indicates smaller errors. The red dots indicate all the event locations in the multi events data.

$677 \times 508 \mathrm{~mm}(72 \times 72 \mathrm{DPI})$ 


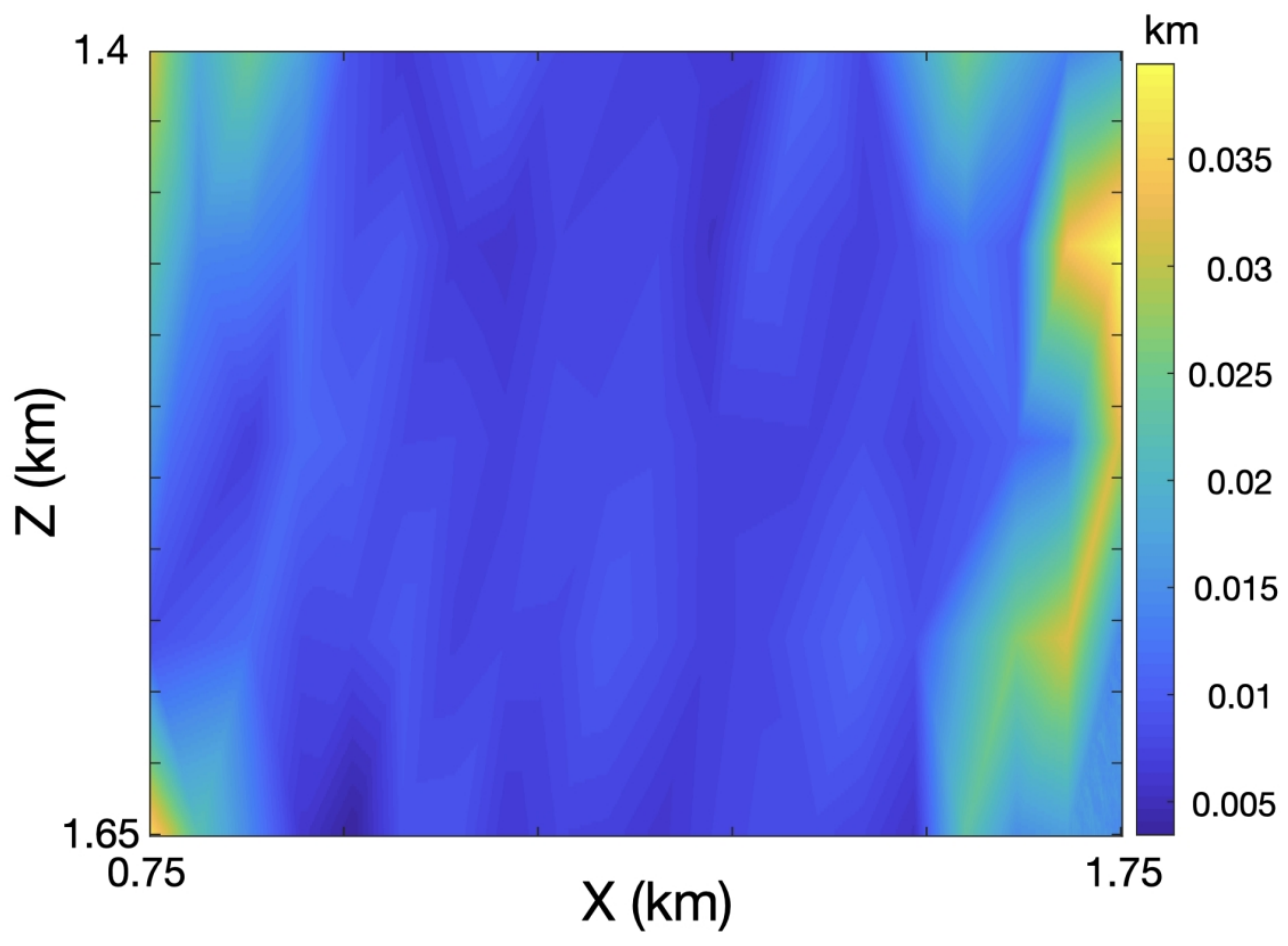

Fig. 14d, Otway model (a) single (c) double, and (e) triple sources cases coordinates $\$ X \backslash \& Z \$$ predictions. Corresponding error maps for (b) single (d) double, and (f) triple sources cases. The yellow color indicates larger errors in space and the blue color indicates smaller errors. The red dots indicate all the event locations in the multi events data.

$677 \times 508 \mathrm{~mm}(72 \times 72 \mathrm{DPI})$ 


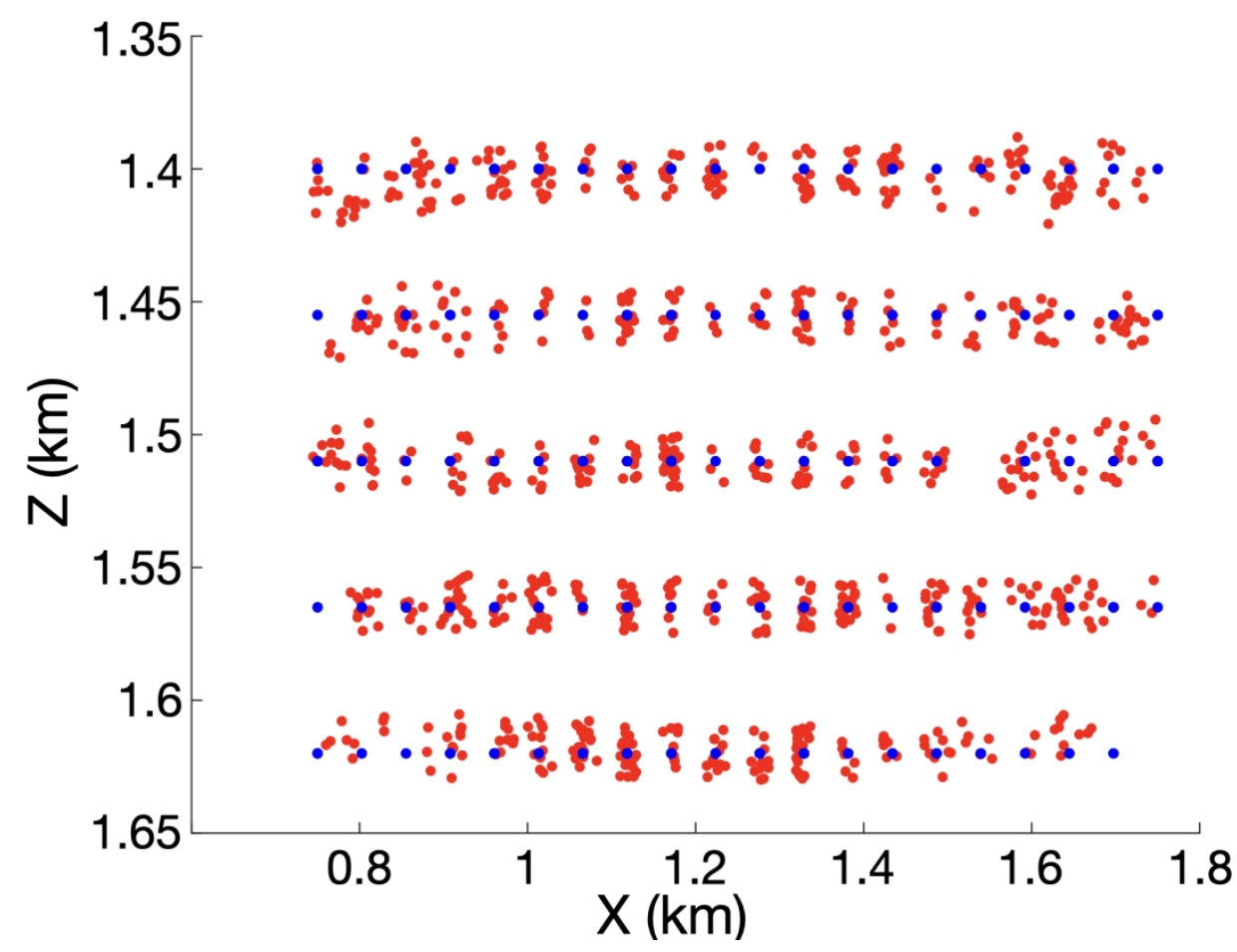

Fig. 14e, Otway model (a) single (c) double, and (e) triple sources cases coordinates $\$ X \backslash \& Z \$$ predictions. Corresponding error maps for (b) single (d) double, and (f) triple sources cases. The yellow color indicates larger errors in space and the blue color indicates smaller errors. The red dots indicate all the event locations in the multi events data.

$677 \times 508 \mathrm{~mm}(72 \times 72 \mathrm{DPI})$ 


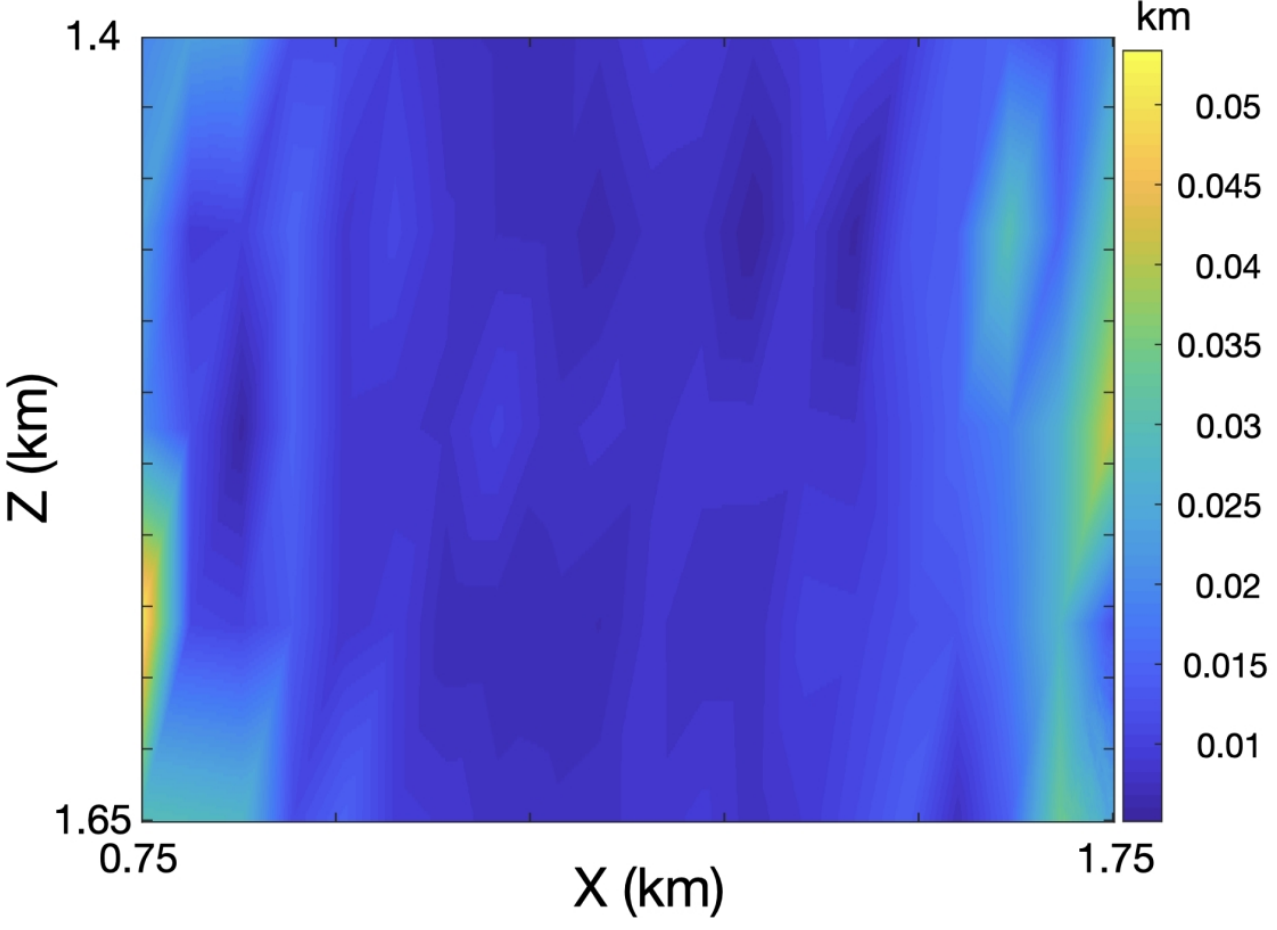

Fig. 14f, Otway model (a) single (c) double, and (e) triple sources cases coordinates $\$ X \backslash \& Z \$$ predictions. Corresponding error maps for (b) single (d) double, and ( $f$ ) triple sources cases. The yellow color indicates larger errors in space and the blue color indicates smaller errors. The red dots indicate all the event locations in the multi events data.

$677 \times 508 \mathrm{~mm}(72 \times 72 \mathrm{DPI})$ 

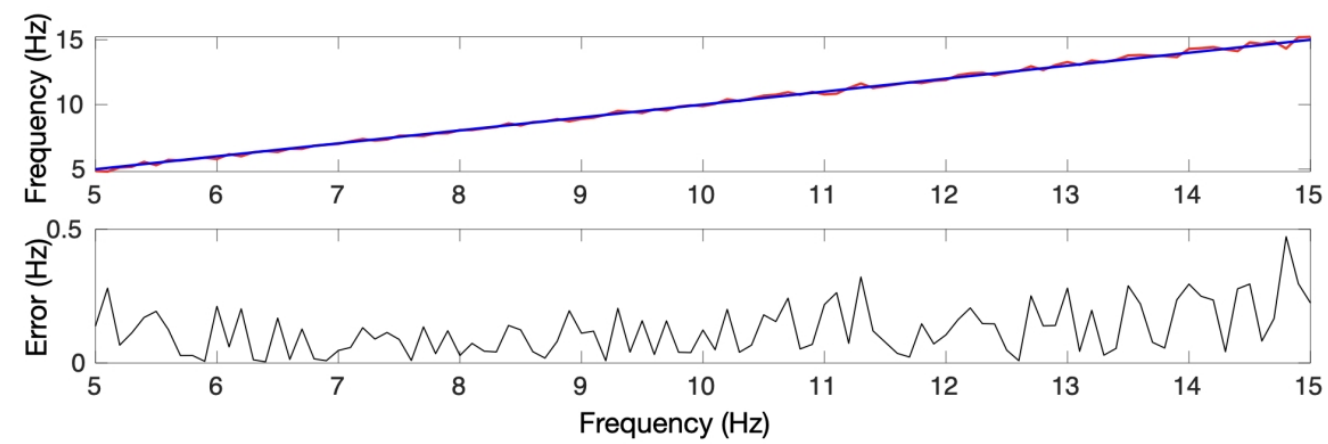

Fig. $15 a$, Predicted source frequencies $\$ F \$$ for (a) single (b) double (c) triple sources cases. $\$ 100 \$$ frequencies are shown for each case. True and predicted labels are shown as the blue and red curves, respectively.

\section{$677 \times 246 \mathrm{~mm}(72 \times 72 \mathrm{DPI})$}



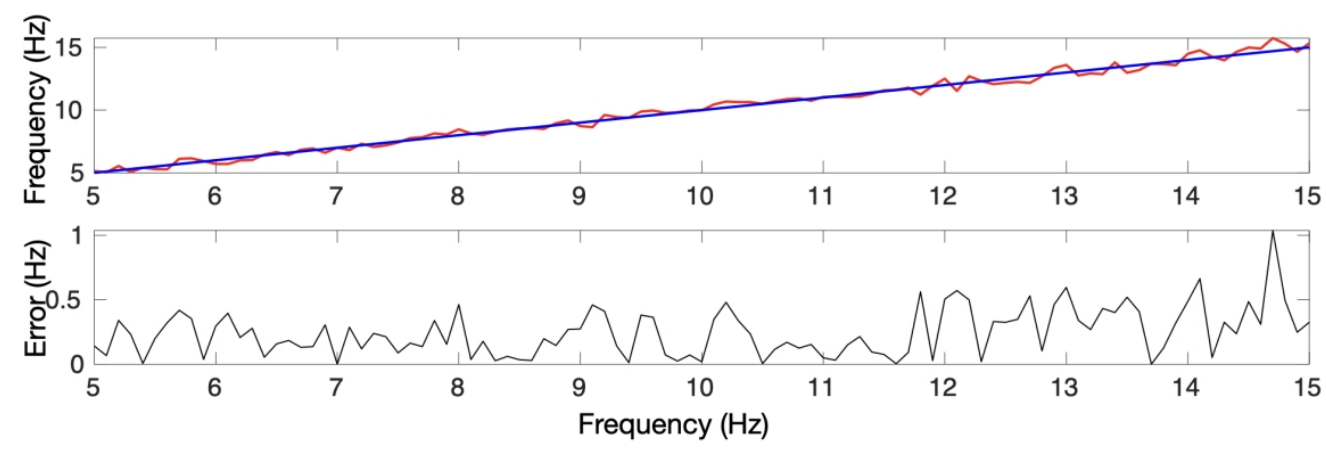

Fig. $15 b$, Predicted source frequencies $\$ F \$$ for (a) single (b) double (c) triple sources cases. $\$ 100 \$$ frequencies are shown for each case. True and predicted labels are shown as the blue and red curves, respectively.

\section{$677 \times 246 \mathrm{~mm}(72 \times 72 \mathrm{DPI})$}



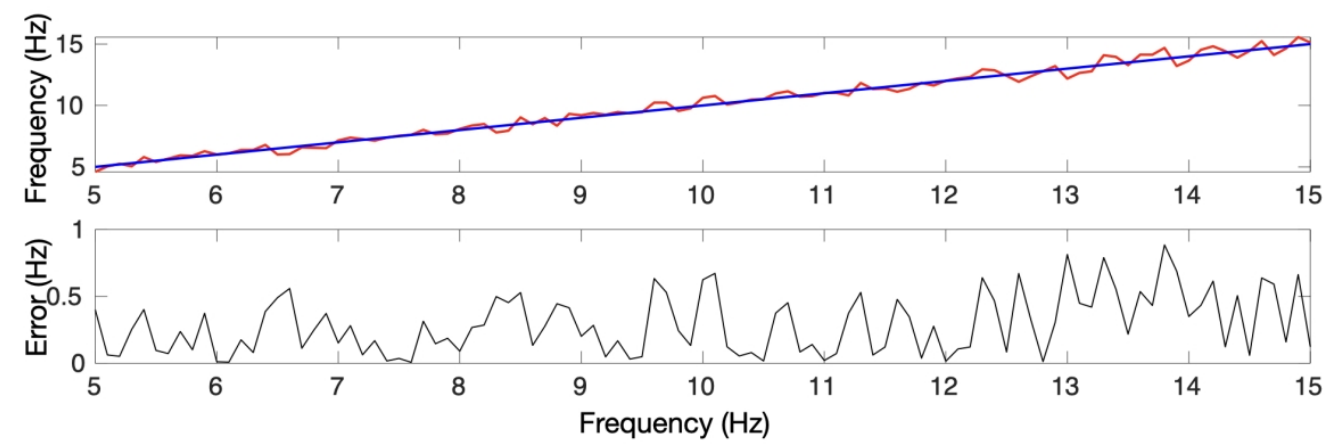

Fig. 15c, Predicted source frequencies $\$ F \$$ for (a) single (b) double (c) triple sources cases. $\$ 100 \$$ frequencies are shown for each case. True and predicted labels are shown as the blue and red curves, respectively.

\section{$677 \times 246 \mathrm{~mm}(72 \times 72 \mathrm{DPI})$}



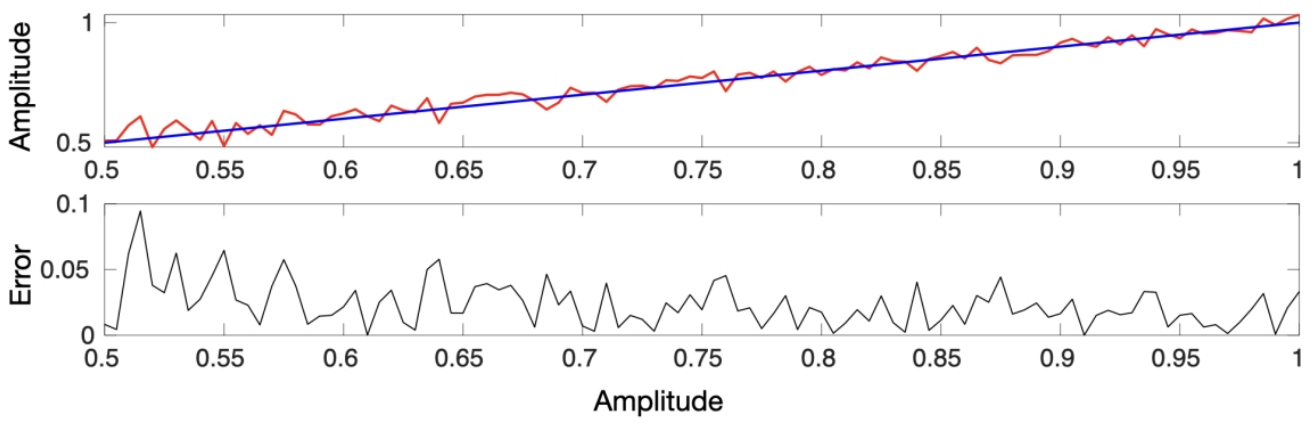

Fig. $16 a$, Predicted source amplitudes $\$ A \$$ for (a) single (b) double (c) triple sources cases. $\$ 100 \$$ amplitudes are shown for each case. True and predicted labels are shown as the blue and red curves, respectively.

\section{$677 \times 246 \mathrm{~mm}(72 \times 72 \mathrm{DPI})$}



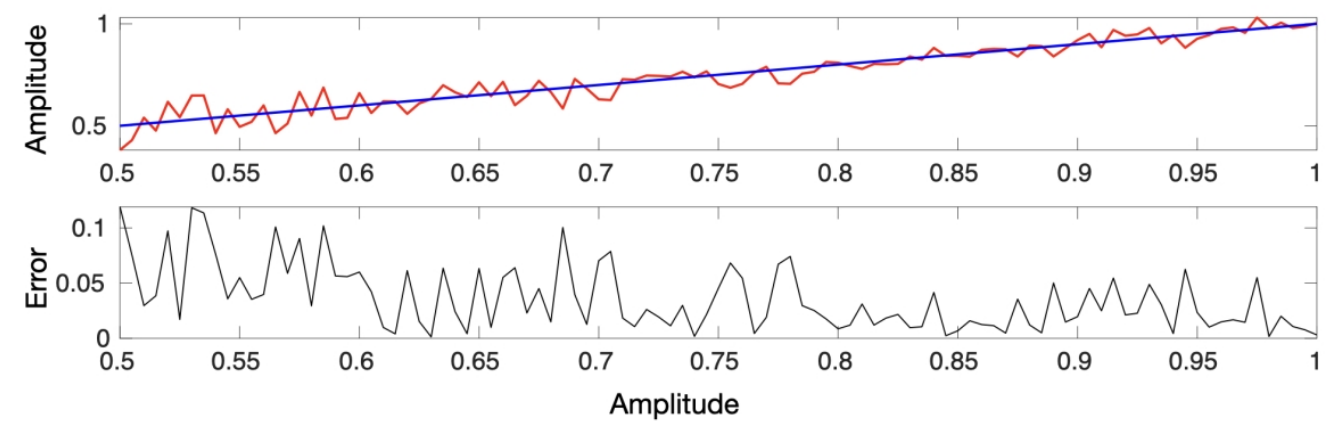

Fig. 16b, Predicted source amplitudes $\$ A \$$ for (a) single (b) double (c) triple sources cases. $\$ 100 \$$ amplitudes are shown for each case. True and predicted labels are shown as the blue and red curves, respectively.

$677 \times 246 \mathrm{~mm}(72 \times 72 \mathrm{DPI})$

This paper presented here as accepted for publication in Geophysics prior to copyediting and composition.

(C) 2021 Society of Exploration Geophysicists 

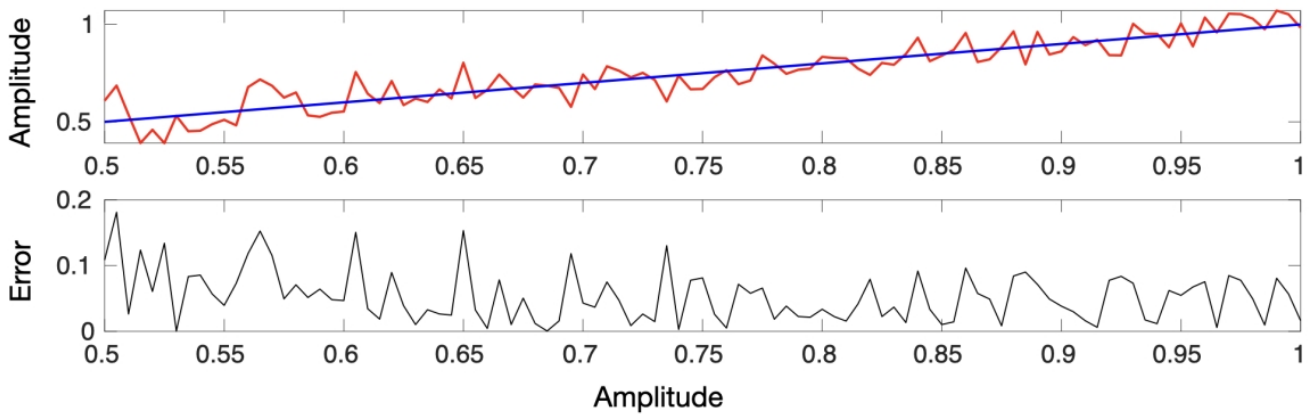

Fig. 16c, Predicted source amplitudes $\$ A \$$ for (a) single (b) double (c) triple sources cases. $\$ 100 \$$ amplitudes are shown for each case. True and predicted labels are shown as the blue and red curves, respectively.

\section{$677 \times 246 \mathrm{~mm}(72 \times 72 \mathrm{DPI})$}




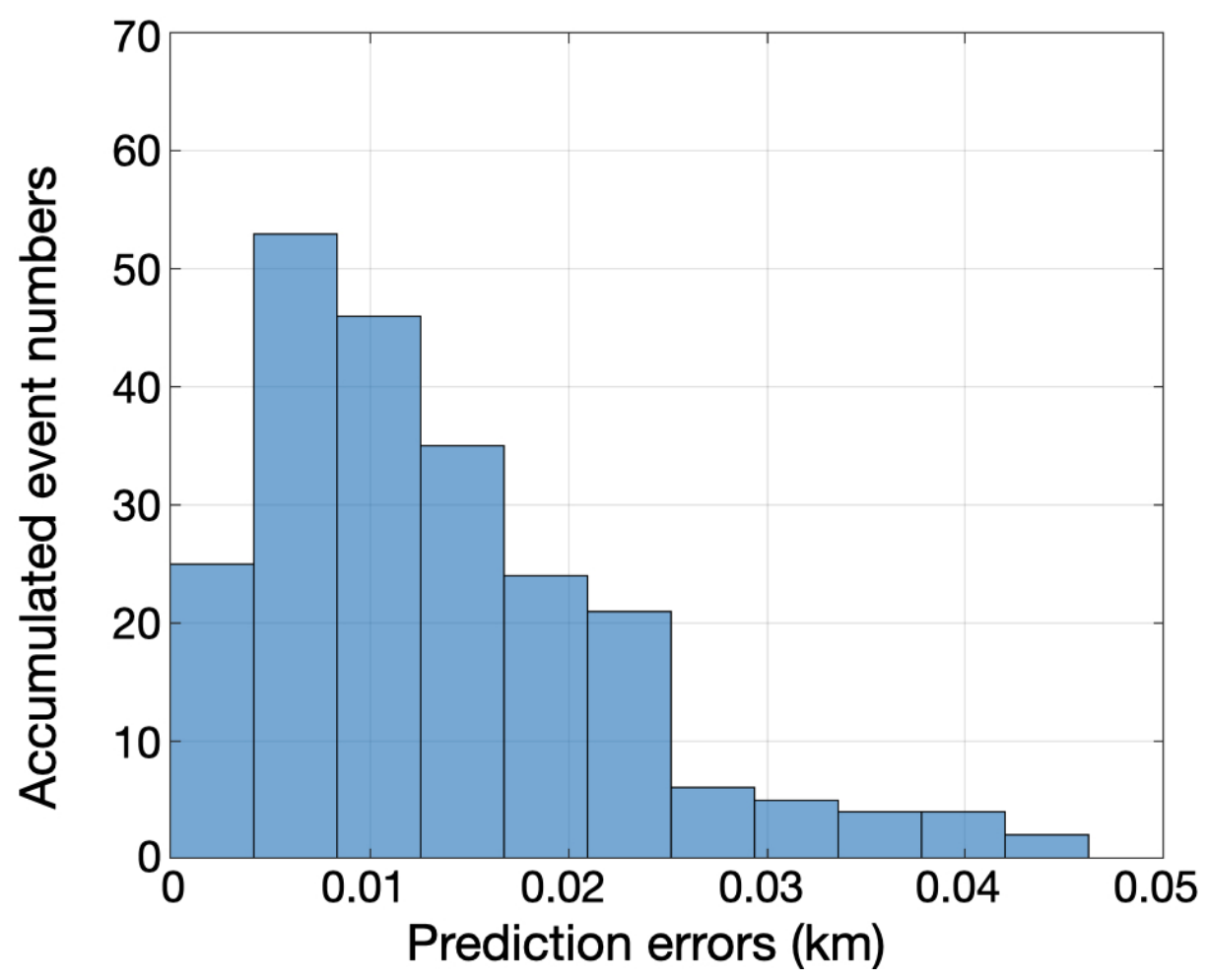

Fig. 17a, (a) Machine learning locating accuracy histogram verses. (b) traditional time-reversal imaging accuracy histogram.

$361 \times 270 \mathrm{~mm}(72 \times 72 \mathrm{DPI})$

This paper presented here as accepted for publication in Geophysics prior to copyediting and composition.

(C) 2021 Society of Exploration Geophysicists 


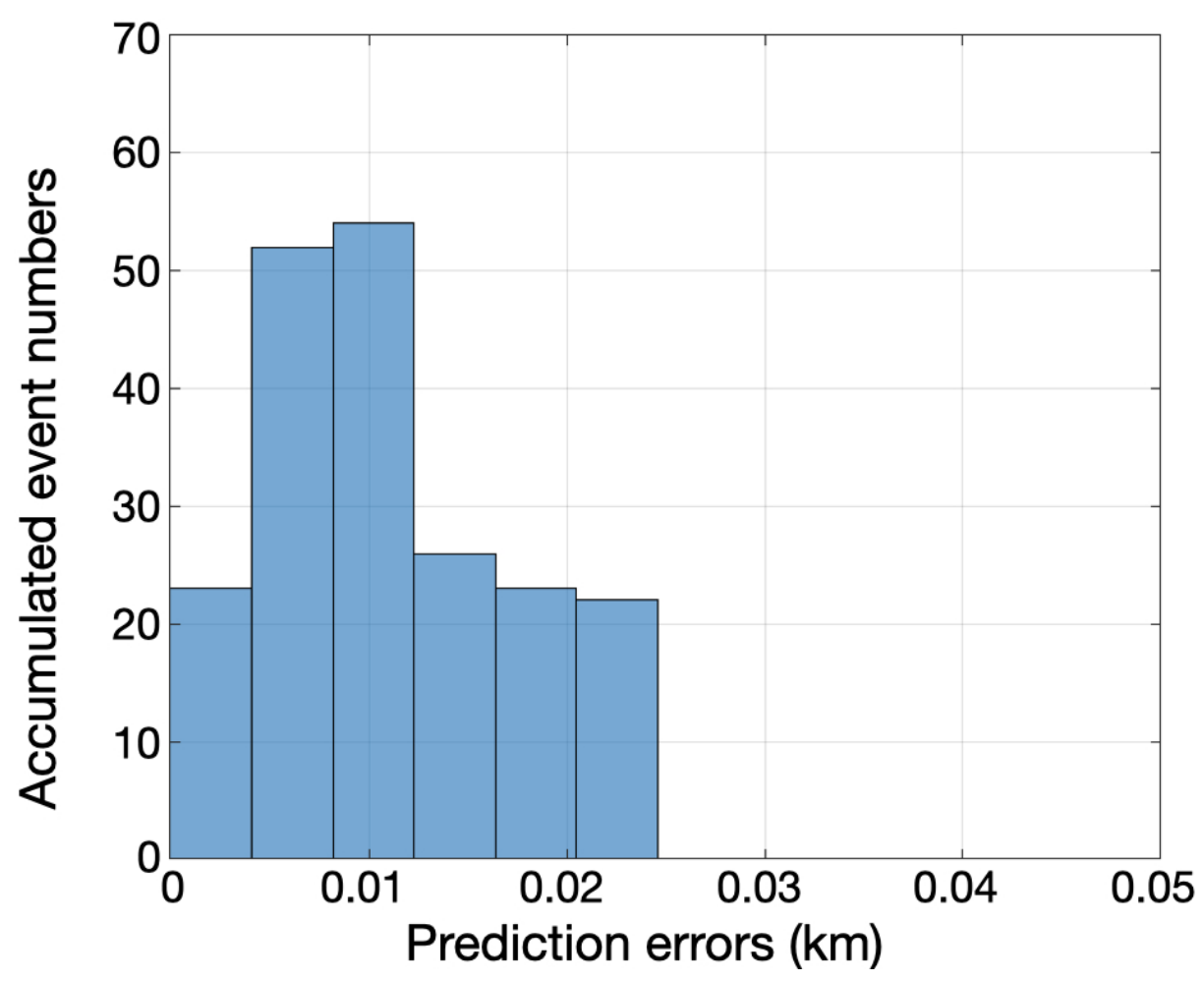

Fig. 17b, (a) Machine learning locating accuracy histogram verses. (b) traditional time-reversal imaging accuracy histogram.

$361 \times 270 \mathrm{~mm}(72 \times 72 \mathrm{DPI})$

This paper presented here as accepted for publication in Geophysics prior to copyediting and composition. (C) 2021 Society of Exploration Geophysicists 
DATA AND MATERIALS AVAILABILITY

Data associated with this research are available and can be obtained by contacting the corresponding author. 